\title{
Future Muon Source Possibilities at the SNS
}

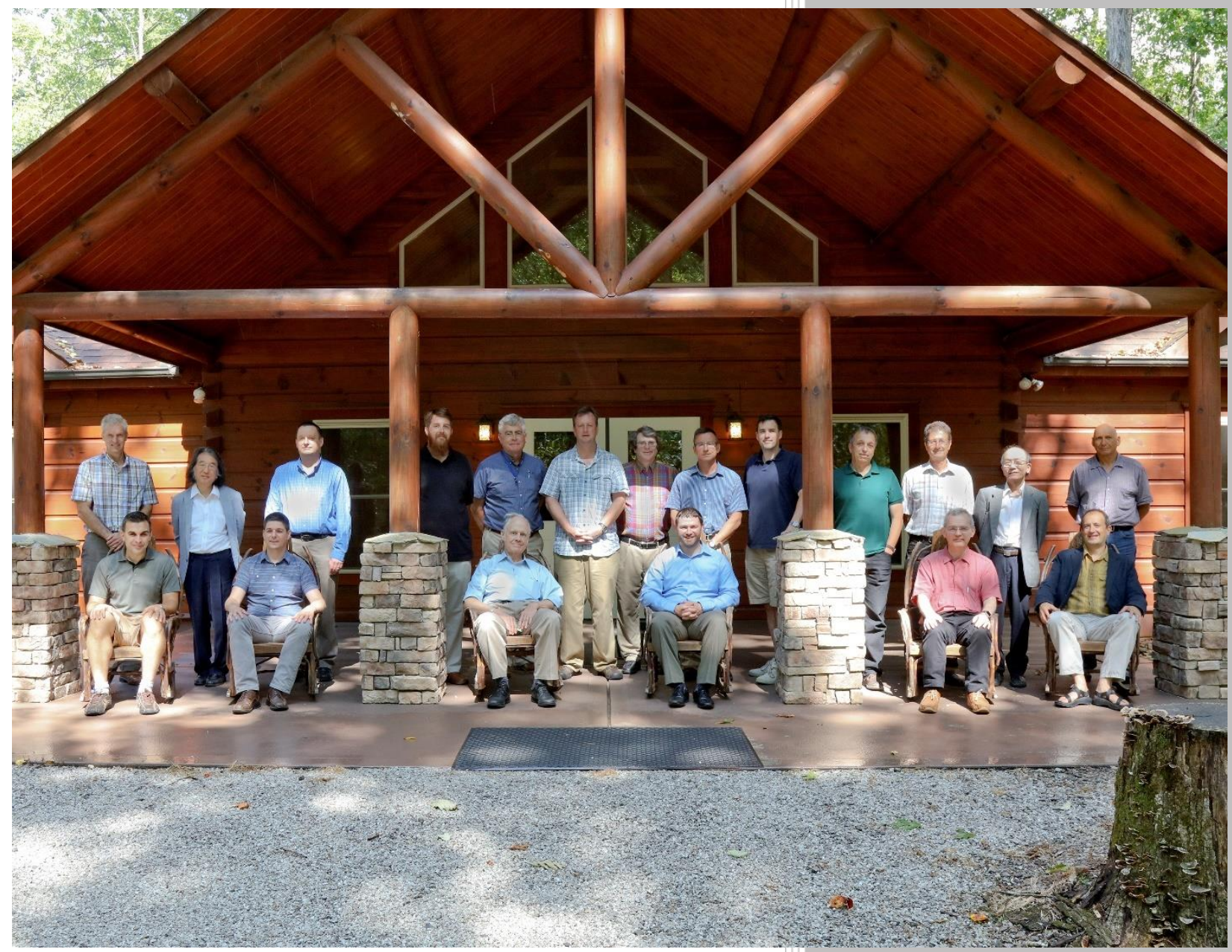

Gregory J. MacDougall Travis J. Williams

Date: March 28, 2017 


\section{DOCUMENT AVAILABILITY}

Reports produced after January 1, 1996, are generally available free via US Department of Energy (DOE) SciTech Connect.

Website http://www.osti.gov/scitech/

Reports produced before January 1, 1996, may be purchased by members of the public from the following source:

National Technical Information Service

5285 Port Royal Road

Springfield, VA 22161

Telephone 703-605-6000 (1-800-553-6847)

TDD 703-487-4639

Fax 703-605-6900

E-mail info@ntis.gov

Website http://www.ntis.gov/help/ordermethods.aspx

Reports are available to DOE employees, DOE contractors, Energy Technology Data Exchange representatives, and International Nuclear Information System representatives from the following source:

Office of Scientific and Technical Information

PO Box 62

Oak Ridge, TN 37831

Telephone 865-576-8401

Fax 865-576-5728

E-mail reports@osti.gov

Website http://www.osti.gov/contact.html

This report was prepared as an account of work sponsored by an agency of the United States Government. Neither the United States Government nor any agency thereof, nor any of their employees, makes any warranty, express or implied, or assumes any legal liability or responsibility for the accuracy, completeness, or usefulness of any information, apparatus, product, or process disclosed, or represents that its use would not infringe privately owned rights. Reference herein to any specific commercial product, process, or service by trade name, trademark, manufacturer, or otherwise, does not necessarily constitute or imply its endorsement, recommendation, or favoring by the United States Government or any agency thereof. The views and opinions of authors expressed herein do not necessarily state or reflect those of the United States Government or any agency thereof.

Approved for public release. Distribution is unlimited. 
Neutron Sciences Directorate

Report from the

Workshop on Future Muon Source Possibilities at the SNS

September 1-2, 2016

Gregory J. MacDougall

Travis J. Williams

Date Published:

March 28, 2017

Prepared by

OAK RIDGE NATIONAL LABORATORY

Oak Ridge, Tennessee 37831-6283

managed by

UT-BATTELLE, LLC

for the

US DEPARTMENT OF ENERGY

under contract DE-AC05-00OR22725 


\section{CONTENTS}

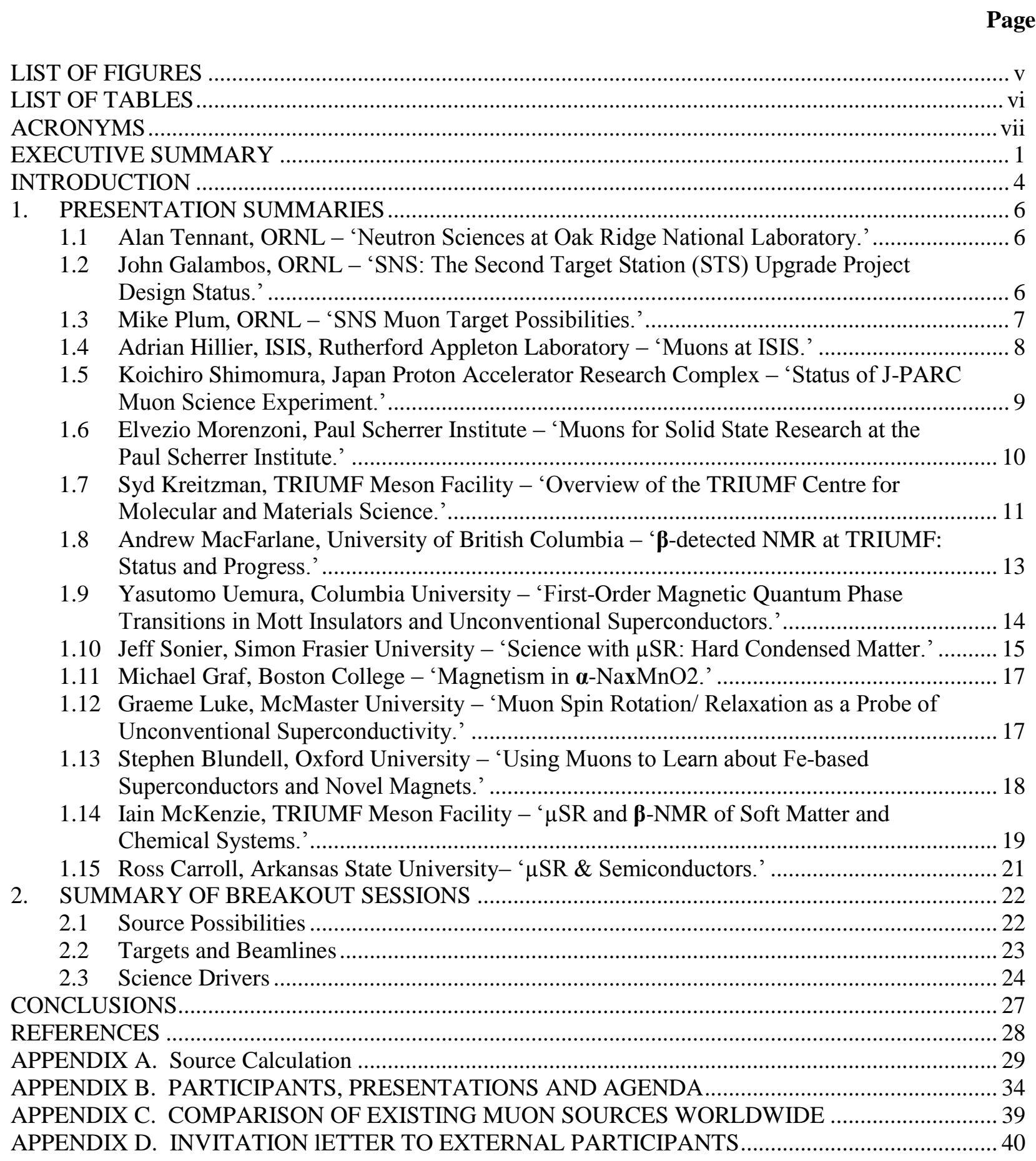




\section{LIST OF FIGURES}

Figure

Figure 1. A schematic of the placement of the proposed SEE facility (see Ref. 1) ................................. 8

Figure 2. The reported distribution of science topics explored in proposals submitted to the

ISIS/RAL muon source.

Figure 3. One idea suggested by Dr. Hillier for a flexible "super- $\mu$ SR" beamline which could increase count rates and potentially also timing resolution from the current generation of $\mu$ SR instruments.

Figure 4. (left) The user numbers at the LMU in PSI over the last ten years. (right) The geographic breakdown of users, using 2014 as an example year.

Figure 5. Schematic of the new detector apparatus being employed in the M9A spectrometer, which mounts SiPM's on the scintillators in the magnet and thus eliminates the need for lightguides.

Figure 6. (left) The asymmetric weak decay of ${ }^{8} \mathrm{Li}^{+}$allows this isotope to be used analogously to $\mu^{+}$ to comment on magnetic properties of materials. (right) Varying the isotope kinetic energy allows a user to choose implantation depth, and student material properties as a function of distance from a surface or interface.

Figure 7. (left) The normalized Bragg peak intensity as a function of pressure in $\mathrm{MnSi}$. (right) The pressure-dependence of the internal field (frequency) and magnetic volume fraction as measured by $\mu$ SR.

Figure 8. A picture and schematic of the MULTI sample holder for performing $\mu$ SR measurements on multiple crystals simultaneously.

Figure 9. (left) Resistivity of $\mathrm{SmB}_{6}$ showing the onset of the bulk insulating state below $10 \mathrm{~K}$. (center) The temperature-dependent relaxation rate in pure and Fe-doped $\mathrm{SmB}_{6}$. The phase transition in the parent compound is smoothed out in the Fe-doped case. (right) The $\mu$ SR spectrum as a function of field, demonstrating the existence of slow magnetic fluctuations.

Figure 10. (left) The structure of $\alpha-\mathrm{NaMnO}_{2}$, showing the muon stopping sites. (center0 the triclinic distortions in the monoclinc bulk structure. (right) The field distribution based on the calculated stopping sites.

Figure 11. The magnetic field distribution in $\mathrm{V}_{3} \mathrm{Si}$ at $\mathrm{H}=3 \mathrm{~T}$ (left) and $\mathrm{H}=5 \mathrm{~T}$ (right).

Figure 12. DFT calculations quantify the effect of a $\mu^{+}$charge on the local crystal field environment in $\mathrm{Pr}_{2} \mathrm{Sn}_{2} \mathrm{O}_{7}$ (left). Similar analysis allows for quantitative analysis of $\mu \mathrm{SR}$ data from on a number of 227 pyrochore systems (right). Adapted from [4].

Figure 13. Example $\mu$ SR data on a particular cholesterolic liquid crystal. (left) A schematic of the cholesterol molecule, showing two muon bonding sites. (center) Time-integrated $\mu \mathrm{SR}$ showing two distinct nuclear resonances. (right) Temperature dependence of this data reveals the presence of two distinct phases: and isotropic phase (I) at high temperatures, and a "narrowing phase" $(\mathrm{N})$ where the molecules wobble around a specific axis thus reducing the width of the resonance line. Adapted from [5].

Figure 14. A recent rf-field resonant $\mu$ SR spectrum from which authors inferred the existence of two shallow muonium acceptor sites in an alloy of silicon and germanium. From [6].

Figure 15. (left) The intensity of the proposed laser pulse as a function of time, with $30 \mathrm{~ns}$ at peak intensity. (right) The resulting time structure of the muon pulse, resulting from the convolution of the laser pulse shape with the pion lifetime. 


\section{LIST OF TABLES}

Table

Table 1. The operational parameters of the SNS accelerator after the PPU project. ............................... 30

Table 2. Name, location and facility type of the 4 muon sources around the world................................. 33

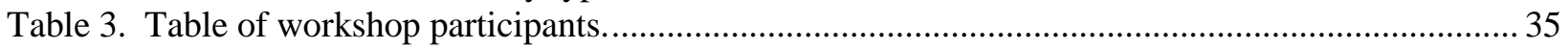

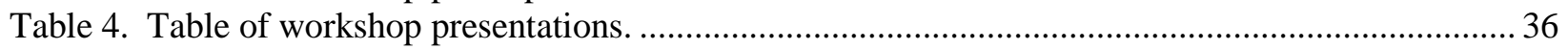




\section{ACRONYMS}

\begin{tabular}{|c|c|}
\hline BES & Basic Energy Sciences \\
\hline CD-1 & Conceptual Design stage 1 \\
\hline DOE & Department of Energy \\
\hline ESS & European Spallation Source \\
\hline FAA & Federal Aviation Administration \\
\hline FTS & First Target Station \\
\hline $\mathrm{GeV}$ & Giga electron volt $\left(10^{9} \mathrm{eV}\right)$ \\
\hline $\mathrm{H}^{-} / \mathrm{H}^{0} / \mathrm{H}^{+}$ & Hydrogen with $2 / 1 / 0$ electrons \\
\hline HFIR & High Flux Isotope Reactor \\
\hline $\mathrm{Hz}$ & $\operatorname{Hertz}\left(\mathrm{s}^{-1}\right)$ \\
\hline ISIS & Rutherford Appleton Laboratory \\
\hline J-PARC & Japan Proton Accelerator Research Complex \\
\hline Linac & Linear Accelerator \\
\hline $\mathrm{mA}$ & milli Amp $\left(10^{-3} \mathrm{~A}\right)$ \\
\hline $\mathrm{MeV}$ & Mega electron volt $\left(10^{6} \mathrm{eV}\right)$ \\
\hline $\mathrm{MHz}$ & Megahertz $\left(10^{6} \mathrm{~Hz}\right)$ \\
\hline MW & Mega Watt $\left(10^{6} \mathrm{~W}\right)$ \\
\hline ns & nanosecond $\left(10^{-9} \mathrm{~s}\right)$ \\
\hline $\mathrm{NScD}$ & Neutron Sciences Directorate \\
\hline ORNL & Oak Ridge National Laboratory \\
\hline PPU & Proton Power Upgrade \\
\hline PSI & Paul Scherrer Institute \\
\hline QCMD & Quantum Condensed Matter Division \\
\hline SEE & Single Event Effects \\
\hline SNS & Spallation Neutron Source \\
\hline STS & Second Target Station \\
\hline $\mathrm{THz}$ & Terahertz $\left(10^{9} \mathrm{~Hz}\right)$ \\
\hline TRIUMF & TRIUMF Meson Facility \\
\hline US & United States \\
\hline$\beta$-NMR & $\beta$-detected Nuclear Magnetic Resonance \\
\hline$\mu \mathrm{s}$ & Microsecond $\left(10^{-6} \mathrm{~s}\right)$ \\
\hline$\mu \mathrm{SR}$ & Muon Spin Relaxation/Rotation/Resonance \\
\hline
\end{tabular}




\section{EXECUTIVE SUMMARY}

The workshop "Future Muon Source Possibilities at the SNS" was held September 1-2, 2016 at Oak Ridge National Laboratory. The workshop aimed to examine the technical feasibility and scientific need to construct a $\mu$ SR and/or $\beta$-NMR facility at the SNS. During the course of the workshop it became evident that recently developed technology could enable the development of a world leading pulsed muon source at SNS, without impacting the neutron science missions of the SNS. The details are discussed below.

Muon Spin Rotation/Relaxation/Resonance $(\mu \mathrm{SR})$ is a technique that involves using spin-polarized muons implanted in a material to provide extremely sensitive measurements of the static and dynamic properties of the local magnetic field distribution within the sample. $\beta$-detected Nuclear Magnetic Resonance $(\beta-\mathrm{NMR})$ is a related technique that employs spin-polarized ${ }^{8} \mathrm{Li}$ ions in the place of muons. These techniques have led to important results in condensed matter physics, chemistry and semiconductor physics, among other fields. In the study of magnetism especially, this technique is highly complementary to neutron scattering: where neutrons provide bulk measurements in reciprocal space with fluctuations on the timescale of nanoseconds (THz), $\mu \mathrm{SR}$ is a local, real space probe, sensitive to microsecond-scale fluctuations (MHz). The two techniques share a common user base, and for this reason 3 of the 4 existing $\mu$ SR facilities in the world are co-located with neutron sources (PSI, ISIS and J-PARC). The glaring exception is in North America, where the sole muon source is located at the meson accelerator laboratory TRIUMF in Vancouver, Canada. The United States has never developed a similar user facility. To address this national shortcoming, there have been several efforts in recent years to assess the feasibility of building a US facility for $\mu$ SR, including a conversation during the construction of the first target station (FTS) of the SNS (2000), and more recently in the context of ProjectX at FermiLab (2013) and the Transformative Hadron Beamlines initiative at Brookhaven National Laboratory (2014). At ORNL, recent successes at the SNS, along with the ongoing PPU and STS expansion projects, have motivated a larger conversation about future science possibilities at the lab. These conditions and the clear national interest have motivated the current workshop to reassess the feasibility of a $\mu$ SR source co-located with the SNS.

The workshop brought together 15 invited researchers from universities and existing $\mu$ SR facilities, along with 8 ORNL staff with detailed knowledge of the SNS accelerator systems, the existing neutron program, and ongoing discussion of the PPU and STS projects. The goals of the workshop were to discuss the scientific challenges that can be addressed by neutrons together with a muon / ion source, the technical requirements for building such a facility, and the perspective of the $\mu$ SR user community on their desire for such a source. The discussions during the workshop were productive, touching on several topics including the state of the $\mu \mathrm{SR}$ community, high-impact science, complementarity with neutron scattering, and muon source possibilities. Particularly promising were discussions surrounding emergent plans for a unique and world leading pulsed muon source at the SNS that could co-exist with the neutron program. Main conclusions of the workshop were as follows:

- $\mu S R$ is a mature probe of condensed matter which continues to produce high-impact science. Measurements using the $\mu$ SR technique have been able to solve outstanding problems in quantum magnetism, chemistry, superconductivity, and semiconductor physics. In studies of magnetism, the sensitivity of $\mu$ SR to minute magnetic fields is leveraged to measure ordered moments as small as $10^{-3} \mu_{\mathrm{B}}$ and emergent local fields associated with novel states breaking timereversal symmetry. Furthermore, the local nature of $\mu \mathrm{SR}$ allows it to be used to detect short range magnetic correlations and to directly determine ordered volume fractions- a strongly complementary measurement to neutron scattering. In superconductors, $\mu \mathrm{SR}$ has been shown to 
be an extremely sensitive measurement of magnetic penetration depth. This absolute measure of the penetration depth is regularly used to calculate the superfluid density as a function of temperature, allowing for strong statements about the nature of the superconducting gap symmetry. For applications to chemistry, it is important to note that muons are long-lived enough to capture an electron, forming muonium. The muonium particle then can then simulate hydrogen and can be used to probe chemical reaction rates. The energy scales of $\mu \mathrm{SR}$ also make it wellsuited to measuring hyperfine coupling constants and level-crossing resonances in chemical systems. In the study of semiconductors, muon hopping rate is probed, which allows for a sensitive measure of quantum diffusion processes, as well as the acceptor/donor state properties on the nanoscale.

- It was strongly emphasized that the co-location of $\mu \mathrm{SR}$ and neutron sources is mutually beneficial to both communities. The international workshop attendees pointed out that three of the four active $\mu$ SR facilities (ISIS, J-PARC and PSI) are co-located with neutron sources, with which they often shared user programs. It was estimated that $10-15 \%$ of neutron users at colocated sources also use $\mu$ SR to study their materials. This complementarity has been strongly utilized by researchers outside of the United States, but also US researchers, albeit on a less frequent basis due to the need for international travel. Several presenters pointed to recent and active collaborations with US neutron scatterers, indicating a growing interest in $\mu$ SR from the US neutron community. Moreover, there has been a recent increase in publications that make use of both techniques, allowing for more impactful conclusions than either technique could have supported alone. It is expected that co-locating muon and neutron sources in the United States would facilitate a large expansion in the $\mu \mathrm{SR}$ user base.

- There have been several recently-developed technologies and routes of development that can be leveraged in a new $\boldsymbol{\mu S R}$ facility. These technologies would fit very well into the technical portfolio of ORNL and the other DOE/BES national laboratories, and will be important in overcoming current limitations of the $\mu$ SR technique. This would position a $\mu$ SR facility at ORNL to have world-leading measurement capabilities. These include RF-techniques that can be used to provide an order of magnitude improvement on timing resolution when measuring in high applied magnetic fields- a particular problem in a technique like $\mu$ SR that makes use of charged particles. In the measurements of semiconductors, the ability to do in-situ optical measurements would increase the capabilities of $\mu \mathrm{SR}$ measurements, while other in-situ techniques can be used for pump-probe and out-of-equilibrium measurements. The development of segmented detectors for $\mu \mathrm{SR}$ measurements has been recently proposed as a means of providing enhanced spatial resolution in measurements and could be employed to develop new muon microscopy facilities. Finally, as with neutron beamlines, $\mu \mathrm{SR}$ measurements have increasingly high demands for extreme sample environments: temperature extremes, high magnetic fields and high pressures. The existing DOE/BES system is well-positioned to address these technical challenges at a cutting-edge $\mu$ SR facility.

- Several possibilities for the location and nature of the muon source at the SNS were considered at the workshop, with one clearly superior than the others:

- The first possibility was to place a muon target in the primary proton beam, prior to the beam entering the FTS or STS buildings. This was one method that was considered during the muon source assessment prior to the construction of the SNS, and is the method currently used at ISIS, PSI and J-PARC. This possibility was rejected for two reasons: its impact on neutron production and the timing structure of the proton beam. Addressing the former point, it was shown that PSI and ISIS lose $\sim 30 \%$ of the protons that would go to neutron production with the muon target in the incident beam. Of this loss, only $2 \%$ of the protons are used to produce muons, while the other $\sim 28 \%$ are lost 
from scattering off the target. On the latter point, the $1 \mathrm{~ms}$ pulse is far too long for muon production and could not be suitably modified to the desired muon timing structure without significant losses in beam intensity. The ideal muon pulse for $\mu$ SR measurements is approximately $30 \mathrm{~ns}$, due to the pion lifetime.

- The second possibility that was considered was to utilize the protons that are currently being directed to the beam dump. This has the advantage that it has no impact on neutron production, but the beam would still have the unfavorable timing structure. The pulse can be made useful for $\mu$ SR by chopping or splitting the beam, however such beam manipulations severely impact the flux. It was decided that the flux of a muon beam using protons from the beam dump would not be high enough to justify the construction of a $\mu$ SR facility. However, the timing structure of a $\beta$-NMR experiment does not suffer these limitations, and so the protons going to the beam stop could be used to create a world-class $\beta$-NMR facility.

- The last source possibility was developed and explored at the meeting, and involves the use of laser-stripping to take extremely short proton pulses from the beam immediately prior to entering the accumulator ring. This idea had been previously proposed for the SEE Systems Test Facility proposed for use by the FAA. This technology has been previously demonstrated, and it could be used to create a proton pulse of any duration with $\sim 70-90 \%$ stripping efficiency. The workshop attendees refined this design and concluded that such a method of producing a muon beam would have negligible impact on neutron production $(0.15 \%$ of the protons would be diverted), would have the best resolution and highest flux of any pulsed muon source in the world. The existing infrastructure of accelerator systems, personnel and technologies means that this worldleading muon source could be constructed with substantial reductions in time, money and technological development relative to a stand-alone facility. 


\section{INTRODUCTION}

Muon Spin Rotation/Relaxation/Resonance $(\mu S R)$ is a technique that involves using spin-polarized muons implanted in a material to provide extremely sensitive measurements of the static and dynamic properties of the local magnetic field distribution within the sample. Proton beams are directed into a low-Z target (typically Carbon or Beryllium) to produce pions. The pions decay with a mean lifetime of $26 \mathrm{~ns}$ via the weak interaction into a muon (or antimuon) and an anti-muon neutrino (or muon neutrino). Muons produced from pions at rest (residing on or near the surface of the target) are known as surface muons, and are the most commonly used muons for $\mu$ SR experiments. The muon carries most of the momentum from this decay, but will stop in fairly thin samples, with a stopping range of $\sim 120 \mathrm{mg} / \mathrm{cm}^{2}$ in carbon. Conversely, muons produced from pions that have been ejected from the target are known as decay muons. These decay muons are useful because of their higher momentum, resulting in deeper penetration into samples and allowing for measurement of materials in an enclosure or high pressure environment. Finally, beams of low energy muons can be produced by taking beams of surface muons and reducing their momentum. This is currently done successfully by passing the beams through thin sheets of noble gases at low temperatures, but there is a developing effort to slow muons using laser pumping. Due to the sharp drop in the muon flux during the slowing process (4-5 orders of magnitude), a high initial flux is needed to make such beamlines feasible. Beams of low-energy muons have a much shorter stopping distance, allowing measurements of thin films, nanostructures and surface properties.

Because the pion decay is governed by the weak interaction, which violates parity, both the neutrinos and muons produced are exclusively left-handed; that is, their spin is antiparallel to their linear momentum. Thus, the surface muons will be $100 \%$ spin polarized, while decay muons have marginally lower $(>80 \%)$ spin polarization since they have to be separated in-flight from pions and electrons/positrons. Depending on the charge of the pion, either positive or negative muons can be produced and used to perform $\mu \mathrm{SR}$ experiments. However, since negative muons have more complex interactions with the samples being measured, nearly all $\mu$ SR experiments tend to be performed with positively-charged antimuons. For the remainder of the report, "muons" could refer to either negativelyor positively-charged muons, but for practical purposes, we strongly emphasize the use of positivelycharged muons. $\beta$-detected Nuclear Magnetic Resonance ( $\beta-\mathrm{NMR}$ ) is a related technique that employs spin-polarized ${ }^{8} \mathrm{Li}$ ions in the place of muons.

Both techniques have led to important results in condensed matter physics, chemistry and semiconductor physics, among other fields. In the study of magnetism especially, this technique is highly complementary to neutron scattering: where neutrons provide bulk measurements in reciprocal space with fluctuations on the timescale of nanoseconds (THz), $\mu \mathrm{SR}$ is a local, real space probe, sensitive to microsecond-scale fluctuations (MHz). The two techniques share a common user base, and for this reason 3 of the 4 existing $\mu$ SR facilities in the world are co-located with neutron sources (PSI, ISIS and JPARC). The glaring exception is in North America, where the sole muon source is located at the meson accelerator laboratory TRIUMF in Vancouver, Canada. The United States has never developed a competitive facility. To address this national shortcoming, there have been several efforts in recent years to assess the feasibility of building a US facility for $\mu$ SR, including a conversation during the construction of the first target station (FTS) of the SNS (2000), and more recently in the context of ProjectX at FermiLab (2013) and the Transformative Hadron Beamlines initiative at Brookhaven National Laboratory (2014). At ORNL, recent successes at the SNS, along with the ongoing PPU and STS expansion projects, have motivated a larger conversation about future science possibilities at the lab. These conditions and the clear national interest have motivated the current workshop to reassess the feasibility of a $\mu$ SR source colocated with the SNS. 
This report summarizes the outcome of the workshop, "Future Muon Source Possibilities at the SNS" held in the Clinch River Cabin at Oak Ridge National Laboratory (ORNL) on September 1 and 2, 2016. The workshop was designed to consider the feasibility and community interest in the construction of a Muon Spin Rotation/Relaxation/Resonance ( $\mu \mathrm{SR})$ and/or $\beta$-detected Nuclear Magnetic Resonance $(\beta-N M R)$ facility co-located with the Spallation Neutron Source (SNS) as a complementary probe of materials, which would enhance the overall user programs in materials research. The workshop brought together 15 invited researchers from universities and existing $\mu$ SR facilities, along with 8 ORNL staff with detailed knowledge of the SNS accelerator systems, the existing neutron program, and ongoing discussion of the Proton Power Upgrade (PPU) and Second Target Station (STS) projects. The letter of invitation to participants (see Appendix C), explained that the goals of the workshop were to discuss the scientific challenges that can be addressed by neutrons together with a muon / ion source, the technical requirements for building such a facility, and the perspective of the $\mu$ SR user community on their desire for such a source.

The scientific program and agenda was organized by G.J. MacDougall (Illinois) and T.J. Williams (ORNL). The agenda (see Appendix B) consisted of: technical talks outlining the existing facilities at the SNS, along with the proposed PPU and STS projects; an overview of the existing muon facilities at the TRIUMF Meson Facility (TRIUMF, Canada), Rutherford-Appleton Laboratory (ISIS, United Kingdom), Paul Scherrer Institute (PSI, Switzerland) and the Japan Proton Accelerator Research Complex (J-PARC, Japan); scientific presentations on the role of $\mu$ SR in condensed matter physics, chemistry and semiconductor physics; and breakout sessions aimed at discussing different possibilities for a putative muon source at the SNS, and necessary conditions such a source must have to address of current and future scientific interest. This report summarizes the presentations from the workshop in Section 1. Section 2 summarizes the conclusions from the breakout sessions, including a preliminary design for a muon source and a comparison of the relative figures of merit to other sources around the world. We then conclude with a summary of main outcomes from the workshop and potential paths forward. 


\section{PRESENTATION SUMMARIES}

\subsection{ALAN TENNANT, ORNL - 'NEUTRON SCIENCES AT OAK RIDGE NATIONAL LABORATORY.'}

Dr. Tennant, Chief Scientist, Neutron Scattering Sciences Directorate (NScD), provided an overview of the neutron scattering facilities and program at ORNL. He outlined the two current neutron sources, the High Flux Isotope Reactor (HFIR) and the Spallation Neutron Source (SNS). HFIR was initially constructed to meet the need for production of transuranic isotopes, but its core mission today is scientific studies that utilize neutron scattering. While still a significant source of isotope production (primarily Cf252) and being used for neutron irradiation and activation analysis, the 12 neutron scattering instruments in the user program at HFIR provide the primary scientific output of the facility. This is due to the high neutron flux of both thermal and cold neutrons. The SNS is a complementary source, being the most powerful accelerator-based neutron source in the world. While being designed as a neutron scattering source from its conception, it was designed to be able to access a greater dynamic range of measurements than HFIR. It currently has 17 instruments in the user program, with 2 others in commissioning and another instrument in the design stage.

Dr. Tennant provided several examples of the scientific productivity of the neutron scattering program at ORNL, including measurements of Fe-based superconductors, thermoelectric materials, materials intended for biomass/bioenergy applications, quantum magnets, energy storage materials and thin film heterostructures. He showed that the number of publications generated by the neutron scattering instruments is continuing to increase, as is the oversubscription rate of both facilities. This is evidence of the vital role that neutron scattering plays in materials research, providing information that cannot be obtained by other techniques. This ability is recognized globally, with several recent investments in new or upgraded facilities, including a power upgrade at J-PARC and a new facility being constructed in Europe, the European Spallation Source (ESS). Likewise, ORNL is investing in two projects: an upgrade to the accelerator allowing operation at higher energy and current, called the Proton Power Upgrade (PPU), and the construction of a second target building to house a planned 22 next-generation neutron instruments, called the Second Target Station (STS).

He emphasized that the success of the current and future neutron sources relies on the continued engagement of the scientific community and a long-term vision of how these facilities can address national and international scientific priorities. This is vital to continuing to attract the frontier experiments and the most positive reflection of the facilities' performance.

\subsection{JOHN GALAMBOS, ORNL - 'SNS: THE SECOND TARGET STATION (STS) UPGRADE PROJECT DESIGN STATUS.'}

Dr. Galambos is the Director of the Spallation Neutron Source Second Target Station Project. He provided an overview of the STS and PPU projects, which have been separated organizationally, but are both required for the advancement of neutron science at ORNL. He described the increased power capabilities that will be derived from the PPU project, including upgrading from 1.4 to $2.8 \mathrm{MW}$, increasing the neutron flux at the FTS from 1.4 to $2.0 \mathrm{MW}$; the additional $800 \mathrm{~kW}$ will be allocated to the STS. This, combined with cutting-edge technology in beamline and detector technology, will make the STS the world's highest peak brightness neutron source, and will be optimized for cold neutrons. He cautioned that adding a muon target in front of the STS neutron target would have a large impact on the beam power delivered to the neutron target. As an example, he cited the dual muon/neutron source at 
PSI, where $1-2 \%$ of the protons are used to create muons, but the proton flux at the neutron target is reduced $20-30 \%$ due to scattering from the muon target upstream.

While outlining the development plan and timeline, Dr. Galambos emphasized that there was a path towards developing a muon source at ORNL. He particularly emphasized two points: the need for a strong science case and its impact on the STS design and performance. The science case for STS has been strongly put forward through a series of workshops and reports over the past several years. This has engaged the wider user community in many different scientific fields in order to demonstrate the need for a balanced, diverse instrument suite. The science case for a muon source must be similarly demonstrated, in terms of its impact, breadth and productivity. With the design of the STS building and instrument layout essentially completed, a muon source design must be made to work within those parameters. Any impact on the design and performance of the neutron source would require strong justification, and would be one of the primary considerations in the approval process for the muon source. Furthermore, with the CD-1 review for the PPU project targeted for mid-2017, there is a short timeline when a muon source design could be considered. However, the long schedule for the accelerator upgrade construction (likely to last through 2024) provides an ideal opportunity for the modification and construction necessary to create a muon source at the SNS.

\subsection{MIKE PLUM, ORNL - 'SNS MUON TARGET POSSIBILITIES.'}

Dr. Plum, the Accelerator Physics Team Leader in the Research Accelerator at ORNL, provided an overview of the linac and accelerator systems. He described the pulse structure and how the $\mathrm{H}^{-}$pulse from the linac was divided between two beam stops and the injection ring. By design, the ring injection is only $95 \%$ efficient, such that $95 \%$ of the beam goes to the accumulator ring (as protons, $\mathrm{H}^{+}$), $3 \%$ goes to the primary beam stop $\left(\right.$ as $\mathrm{H}^{0}$ ) and the remaining $2 \%$ goes to the secondary beam stop (as $\left.\mathrm{H}^{-}\right)$. Either of these two beams could be diverted to a muon target. However, the pulse going to the muon target would have the same time structure as the pulse from the linac, which is $\sim 1 \mathrm{~ms}$ wide. Using the portion of the beam going to the beam dump would allow for a muon source to be located on the opposite site of the accumulator ring from the neutron targets. This has the advantage of not interfering with the design or performance of the neutron program.

A proposal had been suggested in the past for such a facility, that would be used to test Single Event Effects (SEE), proton damage to electronic components ${ }^{1}$, however it was ultimately not constructed. A schematic of this proposed facility and its placement relative to the SNS linac and accumulator ring is shown in Figure 1 . This facility was designed to be $\sim 10,000$ sq. ft., which would easily accommodate a muon source and beamlines instead. While discussing the example of the SEE facility, it was noted that the facility was not designed to use protons from the beam dump, but to utilize a method of laser-stripping the protons to obtain a very short proton pulse in the SEE beamline(s) ${ }^{\text {Error! Bookmark not defined. }}$. This method as estimated to be $90-95 \%$ efficient and could be used to produce a pulse that was arbitrarily short. This method was suggested as a viable alternative to using the protons from the beam dump, and was subsequently acknowledged as has vastly superior beam characteristics for a muon source. Both options were compared during the later break-out sessions, and the conclusion was reached to investigate the laser stripping as the primary method. For further discussion, see the summary of the breakout sessions in Section 2 and the source calculation in Appendix A. 1 


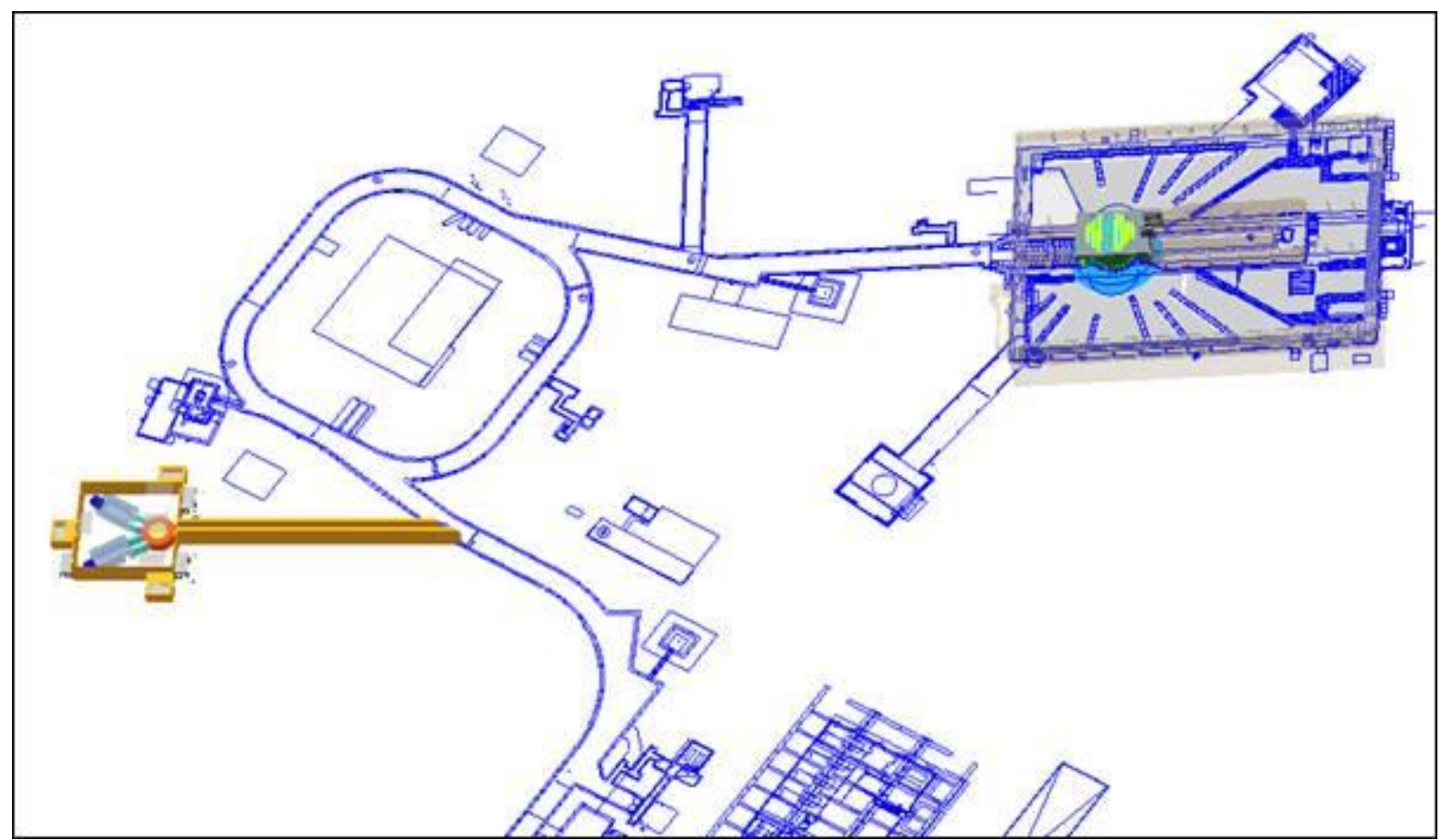

Figure 1. A schematic of the placement of the proposed SEE facility (see Ref. 1).

\subsection{ADRIAN HILLIER, ISIS, RUTHERFORD APPLETON LABORATORY - 'MUONS AT ISIS.'}

Dr. Hillier is the Muon Group Leader for the ISIS facility at Rutherford Appleton Laboratory (RAL) in Oxford, UK, and was slated to present information about the state of the $\mu$ SR program at RAL. Due to last minute personal conflicts, Dr. Hillier was unable to travel to Oak Ridge and his slides were presented to the workshop by Prof. Stephen Blundell. The slides summarized basic information about the ISIS muon source and user base. ISIS is pulsed muon source, created by diverting $4 \%$ of the protons destined for the spallation neutron target. Pulses have $50 \mathrm{~Hz}$ repetition and $70 \mathrm{~ns}$ full-width. Impact on the neutron program is said to be minimal. There are two sides to the $\mu$ SR program at ISIS. The 'EC muon facility', funded by through the European Commission, employs only positive surface muons and has three spectrometers for materials studies. The 'RIKEN-RAL muon facility', operated jointly with the RIKEN lab in Japan, uses both positive and negative muons with variable momentum and operates two spectrometers for materials studies, as well as beam for a low-energy muon development and fundamental physics studies. The existence of a timing structure in the proton beam allows for a large increase in muon flux, limited only by the 'dead time' of positron counters. This latter constraint has led to the extensive use of segmented counters, which increases count rates by a further factor of 5 . Similar efforts should be considered for any high-flux pulsed muon source.

Dr. Hillier noted the science areas explored in the $\mu$ SR program at ISIS, which broadly separates in to studies where muons act as a passive probe (e.g. studies of magnetism, superconductivity, molecular dynamics, charge transport) and studies where the muon is playing an active role (e.g. semiconductors, proton conductors, light particle diffusion). He further gave an interesting breakdown of the size and composition of the user community at RAL, which comprises 60 different groups from 15 countries in Europe. Roughly $30 \%$ of submitted user proposals sought to study inorganic (21\%) or organic (9\%) magnetism, $23 \%$ inorganic $(21 \%)$ or organic (2\%) superconductivity, $11 \%$ elemental analysis, $10 \%$ 
batteries or solar cells, and the remainder covering a host of topics including chemistry, semiconductors, electronic radiation and quantum effects.

The presentation concluded with a discussion of one future path forward for muon pulsed muon facilities. It was acknowledged that the major constraint of such facilities is the timing resolution, which is set by the inherent time width of the incident proton (and thus muon) pulses. Dr. Hillier suggested that resolution can be improved by temporally 'slicing' the muon beam to effectively decrease pulse width, and presented a plan for a "SuperMuSR" beamline at ISIS that could operate in "High Resolution" mode, with $10 \times$ the resolution and $2 \times$ the current count rate, or "High Rate" mode, with $15-20 \times$ the current rate. A survey of the requirements for various $\mu$ SR experiments revealed that some would benefit more from rate and some more from resolution. Thus, it was concluded that there would be a distinct benefit in having a muon facility able to vary from one mode to the other. This possibility was discussed extensively in the context of a potential new source at the SNS.

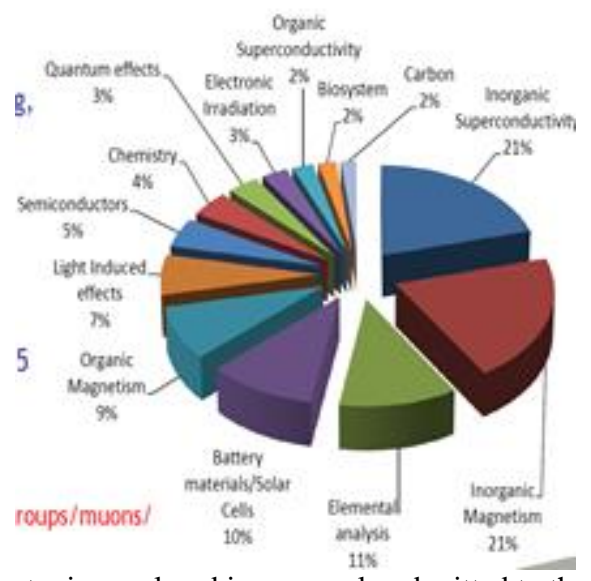

Figure 2. The reported distribution of science topics explored in proposals submitted to the ISIS/RAL muon source.

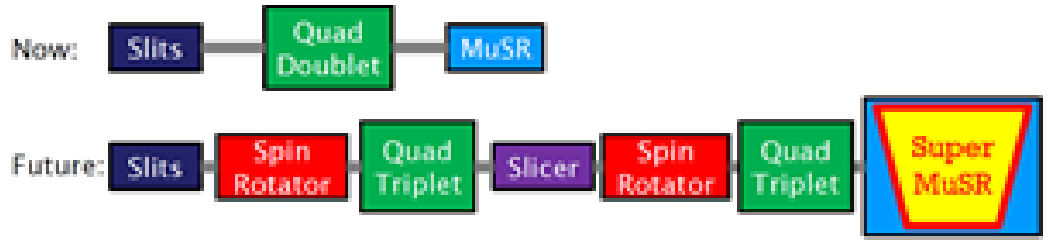

Figure 3. One idea suggested by Dr. Hillier for a flexible "super- $\mu$ SR" beamline which could increase count rates and potentially also timing resolution from the current generation of $\mu$ SR instruments.

\subsection{KOICHIRO SHIMOMURA, JAPAN PROTON ACCELERATOR RESEARCH COMPLEX - 'STATUS OF J-PARC MUON SCIENCE EXPERIMENT.'}

Dr. Shimomura attended as a representative for the Muon Science Establishment (MUSE) in the Material and Life Science Facility (MLF) at the Japan Proton Accelerator Research Complex (J-PARC). He began by laying out the source at J-PARC and the instruments in MUSE. The muon program at JPARC is parasitic to the neutron spallation source, and uses the same $25 \mathrm{~Hz}$ proton beam where each timing cycle contains a pair of $100 \mathrm{~ns}$ wide pulses are separated by 600ns (a "double bunch" structure). Muons are produced using a rotating graphite target, which has been operating since September, 2014 with no reported problems. As designed, the facility will ultimately have four muon beamlines with different purposes, but they are in varying stages of construction. 
Dr. Shimomura discussed the purpose and design specifics of each of the four beams. The most detail was given about the 'D-line', a multi-purpose beam where either surface (30MeV/c) or decay (5$120 \mathrm{MeV} / \mathrm{c})$ muons can be extracted. This beamline was first commissioned in 2014, soon after the graphite source was installed, and is now fully in the user program. Detail was given about spectrometer designs, all of which use segmented detectors, and the newest of which is able to count $200 \mathrm{M}$ events/hour. It was clear that effort has been put into developing proper sample environments, including a recent addition of a dry dilution refrigerator which can be cooled in 1.5 days without need for human intervention. Dr. Shimomura then discussed the 'U-line', a dedicated 'ultra-slow muon' beam with high intensity and high luminosity. This beamline has $10 \times$ the incident flux as the D-line, and muons are slowed to final momenta in the range $0.05-50 \mathrm{keV} / \mathrm{c}$ using laser pumping techniques. The U-line has long been in development, but current milestones have been hit in the past year, and as of 2016, the beam is capable of providing a usable rate of 40 ultra-slow muons/second. Also discussed were the 'S-line', a second surface muon beam that began commissioning in October, 2015, and the 'H-line', which is in planning stages but is to include instruments for muon $g-2$ experiments, muon transmission microscope and $\mathrm{Mu}-\mathrm{HF}$ measurements. Despite the facility being in development stages, Dr. Shimomura gave encouraging user numbers. He stated that the one instrument in the user program, D1, had 108.5 days of operation in 2015 and received 40 proposals for that time. The instrument was a factor of 2 oversubscribed. The user base was $90 \%$ Japanese, with the other $10 \%$ coming mostly from China and Korea, demonstrating a strong role for geographic proximity.

On a final note, Dr. Shimomura mentioned that J-PARC has recently undergone a power upgrade to $1 \mathrm{MW}$, and is in the planning stages for a second neutron/muon target station. The muon program is expected to benefit greatly from this development, with projected muon intensities a factor of 50 greater than the first target station (factor of 10 from $\mathrm{W}$ target, and 5 from beamline capture). The large increase in flux is leading to consideration of new classes of muon instrumentation, including an ultra-highintensity muon beam stroboscope for muon imaging experiments.

\subsection{ELVEZIO MORENZONI, PAUL SCHERRER INSTITUTE - 'MUONS FOR SOLID STATE RESEARCH AT THE PAUL SCHERRER INSTITUTE.'}

Dr. Morenzoni presented the state of the program at the Laboratory for Muon Spin Spectroscopy (LMU) at the Paul Scherrer (PSI) Institut in Villigen, Switzerland. His talk consisted of an overview of the instruments in the LMU program, a summary of scientific output, and select science examples. At PSI, muons are created by diverting a fraction of protons from the spallation neutron target $(0.4 \mathrm{GeV}$, $2.4 \mathrm{~mA}=1.5 \times 10^{16}$ protons/second) towards a rotating graphite target to create quasi-continuous beams of surface $(4 \mathrm{MeV})$, decay $(10-60 \mathrm{MeV})$, and ultra-low energy $(1-30 \mathrm{keV})$ muons. Surface muons are $100 \%$ polarized, and these channels have $10^{7}-10^{9}$ muons/second for condensed matter studies. Decay muons are $80 \%$ polarized, and energies can be chosen in the $\mathrm{MeV}$ range for depth-ranging muons into samples contained in pressure cells or other enclosed environments. Ultra-low energy muons are used for depthdependent studies in the range $2-300 \mathrm{~nm}$ from a sample surface, and LMU supplies $1.9 \times 10^{8} \mathrm{\mu}^{+} / \mathrm{s}$ towards a dedicate low-energy beamline for this purpose.

Dr. Morenzoni describes in detail the six instrument end stations in the LMU program. Each end station has a dedicated spectrometer, and as with other facilities, there was a strong emphasis on the importance of sample environment capabilities. There are four instruments for utilizing surface muons: HTF, a new spectrometer capable of measuring in fields as high as 9T while applying temperatures below $20 \mathrm{mK}$; LTF, a spectrometer dedicated to measurements at dilution refrigerator temperatures in fields below 0.6T; and GPS and DOLLY, two general purpose instruments for performing measurements in fields below $0.6 \mathrm{~T}$ and temperatures above $250 \mathrm{mK}$. GPS and LTF share a single beamline, where an 
electrostatic kicker only sends a muon to a specific instrument when needed (Muons On Request), thus lowering background to levels more commonly associated with pulsed muon sources. LMU also has a dedicated decay muon channel, GPD, used primarily for studies in pressure cells (max pressure of 2.8GPa). A series of impressive examples demonstrated how these capabilities have allowed for impactful science with $\mu$ SR at PSI. This included determination of phase diagrams and demonstration of co-existing superconducting and magnetic volumes in FeAs materials, detection of $70 \mathrm{G}$ time-reversal symmetry breaking fields in the non-centrosymmetric superconductor SrPtAs, contribution to discovery of a spinliquid phase in $\mathrm{Ca}_{10} \mathrm{Cr}_{7} \mathrm{O}_{28}$, and studies of the quantum critical point in quasi-skutteridite $\mathrm{R}_{3} \mathrm{~T}_{4} \mathrm{X}_{13}$ with $\mathrm{T}=\{\mathrm{Ir}, \mathrm{Rh}\}$. Examples concentrated on magnetism and superconductivity, reflecting Dr. Morenzoni's own training, but he made it clear there was also interesting work being performed at LMU in the areas of chemistry, soft-matter, applied physics and semiconductor physics, among other areas.

A significant amount of time was spent discussing the dedicated low-energy $\mu$ SR (LE- $\mu$ SR ) beamline, which for several years has been performing novel and cutting-edge studies of thin-film samples and heterostructures. The LE- $\mu$ SR program at PSI is unique in the world, and uses $1.9 \times 10^{8} \mu^{+} / \mathrm{s}$ on a dedicated surface muon source in conjunction with a series of moderators, kickers and electrostatic fields to create a beam of muons with kinetic energies $0.5-30 \mathrm{keV}$ at a typical rate of $4500 \mu^{+} / \mathrm{s}$ at the sample site. By varying kinetic energy, one can vary the depth from the sample surface a muon will implant. Dr. Morenzoni presented interesting applications of these new techniques, including the measurement of spin diffusion lengths in organic spin valves, observation of a giant proximity effect in $\mathrm{LaCu} 2 \mathrm{O} 4$ multilayers, and measurement of an intrinsic paramagnetic Meissner effects in an oddfrequency superconductors. As the newest item in the $\mu$ SR toolbox, he made clear that it was opening up the technique to new scientific communities.

Overall, a positive picture was painted of state of the user community in the LMU at PSI. Over the past decade, the number of user proposals received at the institution has increased $70 \%$, and in 2015 they received 207 proposals to cover 763 total experiment days. Instruments were all oversubscribed, by factors ranging from 2.0-3.5. About 60 papers are published each year containing data from experiments at LMU, including 10 in journals that are traditionally labelled as "high impact". The user community is $50 \%$ based in Switzerland and 30\% elsewhere in Europe, again reinforcing the huge positive impact of geographic proximity, though Dr. Morenzoni noted that about 5\% of the user base is travelling each year from the United States.
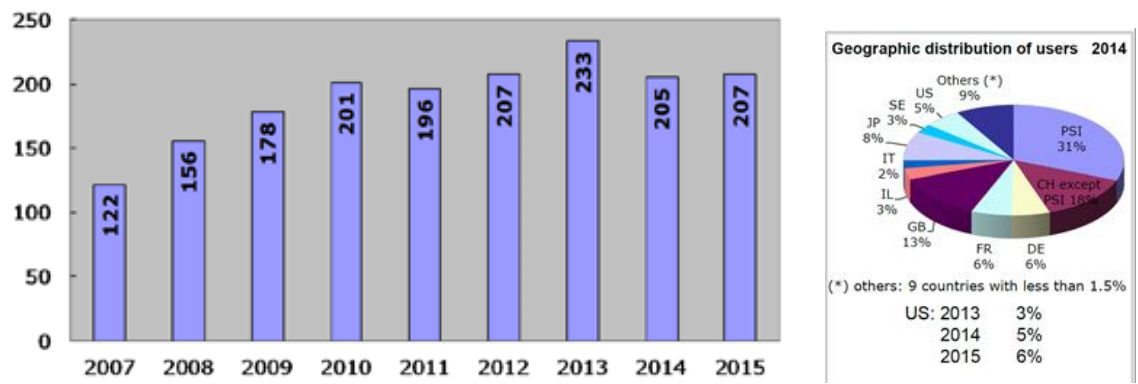

Figure 4. (left) The user numbers at the LMU in PSI over the last ten years. (right) The geographic breakdown of users, using 2014 as an example year.

\subsection{SYD KREITZMAN, TRIUMF MESON FACILITY - 'OVERVIEW OF THE TRIUMF CENTRE FOR MOLECULAR AND MATERIALS SCIENCE.’}

Dr. Kreitzman is the Manager of the Centre for Molecular and Materials Science (CMMS) at the TRIUMF meson facility in Vancouver, Canada, which is the sole $\mu$ SR user program in North America. His talk focused on programmatic elements of the $\mu$ SR and $\beta$-NMR facilities at TRIUMF, giving detailed 
information about the number of staff, beamlines, spectrometers, and the state of various repairs and developments. TRIUMF has 6 scientific staff and 4 technical staff members, funded largely via 5 year contracts. It currently has two operational beamlines in the $\mu$ SR program, each of which supplies a continuous beam of surface muons. The M15 beamline began operation in 1984 and is one of the most productive in the program, though it is beginning to show problems related to its age, reduced rates due to the degradation of permanent magnets in the beamline optics. The other beamline, M20, was recently split into two separate end-stations with a high-frequency "kicker" providing muons to both. The facility also has a third beamline, M9, split into surface muon (M9A) and decay muon (M9B) channels. The decay channel has historically been used for chemistry and high-pressure experiments, but unfortunately it has been out of commission for several years due to a fundamental issue with the alignment of the associated muon target. In addition to the muon facility, the CMMS has in recent years been maintaining a $\beta$-NMR program, which uses particles from the rare isotope facility at TRIUMF to explore thin-films and surface physics. Due to beam availability, the $\beta$-NMR program only operates 5 weeks/year.

The CMMS program at TRIUMF is undergoing an unprecedented period of expansion, and Dr. Kreitzman detailed several ongoing and future developments. In addition to the recent doubling of capacity on M20 from splitting the beam, a Muons on Request setup, is being commissioned and should lower the overall background. On M15, all of the magnet power supplies and heat sinks have been replaced. Detail was given about the newly constructed helium liquefier, which is expected to greatly reduce user costs. One of the two M9 end-stations (M9A) is due to come online in 2017 and should direct a new channel of surface muons towards a versatile 3T spectrometer. In addition, a consortium of university-based research professors have recently submitted a $\$ 10 \mathrm{M}$ proposal to the Canadian Foundation for Innovation to upgrade and expand the decay channel (M9B), and also provide a reliable source of negative mons for fundamental physics and chemistry applications. With the opening of the new ARIEL isotope facility in 2020, the $\beta$-NMR beamlines are set to triple the available beamtime and greatly expand the program.

The functionality of the six different spectrometers in the program was detailed and, as elsewhere, particular emphasis was placed on sample environment capability. Dr. Kreitzman discussed a new highmagnet field spectrometer, NuTime, which has just entered the program and is capable of measuring in fields as high as $\mathrm{H}=7 \mathrm{~T}$ with $1.5 \mathrm{ppm}$ field homogeneity at the sample site. He highlighted the dilution refrigerator, which is capable of cold sample changes, can apply fields of up to 3T, and has base temperature of $12 \mathrm{mK}$. Coming up, he spoke about a new generation of spectrometers which employ active muon collimation and scintillator-mounted $\mathrm{Si}$ photo-multipliers, eliminating light guides and allowing for lower background and timing resolution as small as 50ps. A detailed plan was laid out for new $\beta$-NMR spectrometers and facilities, including the development of a liquid $\mathrm{Mg}$ isotope target argued to be ideal for biological studies. Finally, Dr. Kreitzman detailed the extensive outreach and community expansion efforts being supported by the CMMS program, which aims to raise awareness of $\mu$ SR in the U.S. institutions and broaden in the North American user community in coming years.

Throughout his talk, Dr. Kreitzman spoke positively of the prospect of a muon source of SNS, and specifically pointed to the strong collaborations between $\mu$ SR and American neutron scattering user bases. He mentioned that there are 48 separate U.S. neutron scatters that have been users at TRIUMF in recent years. He also spoke of the complementarity between the continuous and pulsed muon facilities, and suggested such complementarity would extend to the source at TRIUMF and any pulsed facility at ORNL. The SNS, he pointed out, naturally has the timing characteristics and expertise in RF technology to build a world-class Muon Magnetic Resonance program, akin to the program at ISIS, and could build a pulsed facility that would put ORNL on a "Pinnacle of the MuSR World". 


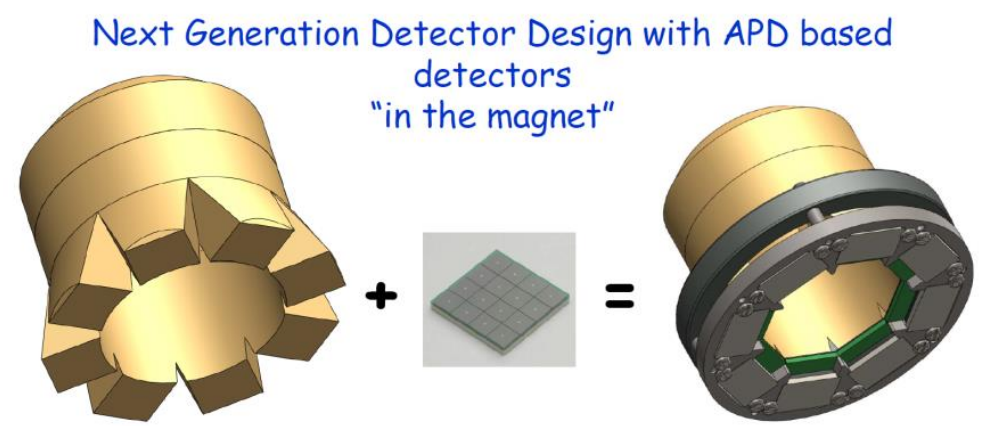

Figure 5. Schematic of the new detector apparatus being employed in the M9A spectrometer, which mounts SiPM's on the scintillators in the magnet and thus eliminates the need for light-guides.

\subsection{ANDREW MACFARLANE, UNIVERSITY OF BRITISH COLUMBIA - ' $\beta$-DETECTED NMR AT TRIUMF: STATUS AND PROGRESS.'}

Dr. MacFarlane, Associate Professor in the Department of Chemistry at the University of British Columbia introduced the technique $\beta$-detected NMR ( $\beta$-NMR), and discussed its scientific impact and future directions for the technique. The $\beta$-NMR program at TRIUMF is unique in the world, and uses the rare-isotope production facility there to create spin polarized beams of radioactive isotopes which are then implanted in materials of interest, evolve in the local field environment and undergo $\beta$-decay. The technique has strong parallels with $\mu \mathrm{SR}$, and obtains similar but distinct pieces of information. The longer decay times of isotopes make them sensitive to fluctuations on the timescale of seconds, rather than microseconds for muons, making them better suited for measurements of spin-lattice relaxations in metals, for example. Further, the low kinetic energies involved allow for controlled implantation depths of isotopes over the range 100-2000 $\AA$, making the technique a natural complement to neutron reflectometry in the study of surface or interface magnetism. Dr. MacFarlane talked about isotope production at TRIUMF, which involves irradiating specially design tantalum targets at temperatures exceeding $2000 \mathrm{C}$ with $500 \mathrm{MeV}$ protons. This process produces high quality beams of isotopes with diameter $4-8 \mathrm{~mm}$ at a rate of $\sim 10^{7}$ ions/second. Ion beams are then actively spin polarized using circularly polarized laser light, and momentum is chosen by maintaining an electric potential difference between the ion target and sample. The most commonly used isotope is ${ }^{8} \mathrm{Li}^{+}$, which is sensitive to electric quadrupoles in addition to spins, but researchers at TRIUMF also have access to spin-only ${ }^{31} \mathrm{Mg}^{2+}$ ions. Typical measurements last $\sim 1 \mathrm{hr}$, comparable to $\mu \mathrm{SR}$.

Though now fully in the user program at TRIUMF, $\beta$-NMR currently runs on a restricted schedule of 5 weeks of beamtime per year. Dr. MacFarlane emphasized that the technique is still in its early days, comparable to the state of $\mu$ SR in the early 1980s, and that much of the early research has focused on finding best operating procedures and basic characterization of spectrometers properties such as backscattering and depth profiles. He proceeded, however, to give examples of important scientific results from recent years. This included measurements of surface effects in $\mathrm{Fe}_{2} \mathrm{O}_{3}$ below the Morin transition, second timescale spin fluctuations in correlated metal $\mathrm{Sr}_{2} \mathrm{RuO}_{4}, \mathrm{Li}+$ diffusion at ionic heterointerfaces, and orbital/spin response in topological insulator $\mathrm{Bi}_{0.9} \mathrm{Sb}_{0.1}$. The newly constructed ARIEL isotope facility at TRIUMF is slated to contain a dedicated $\beta$-NMR beamline.

Dr. MacFarlane communicated that lessons learned at TRIUMF could guide the design and construction of a similar $\beta$-NMR facility at the SNS. For example, he notes that the Ta-foils used to produce ${ }^{8} \mathrm{Li}^{+}$-ions have a 6 -week lifetime, and specially designed targets and protocols would need to be developed to change "hot" targets on a semi-regular basis. Tuning and beamline alignment, currently a 
limiting step at TRIUMF, would be helped by modern technologies. He further emphasized that the main source of errors limiting data are systematic in nature, not statistical, and that beam stability should be emphasized over raw flux in any future design. The $60 \mathrm{~Hz}$ repetition rate and pulse characteristics in the FTS or beam dump which are quite limiting for the design of a muon source, are considered suitable for $\beta$-NMR, to which a $60 \mathrm{~Hz}$ timing structure would appear quasi-continuous.
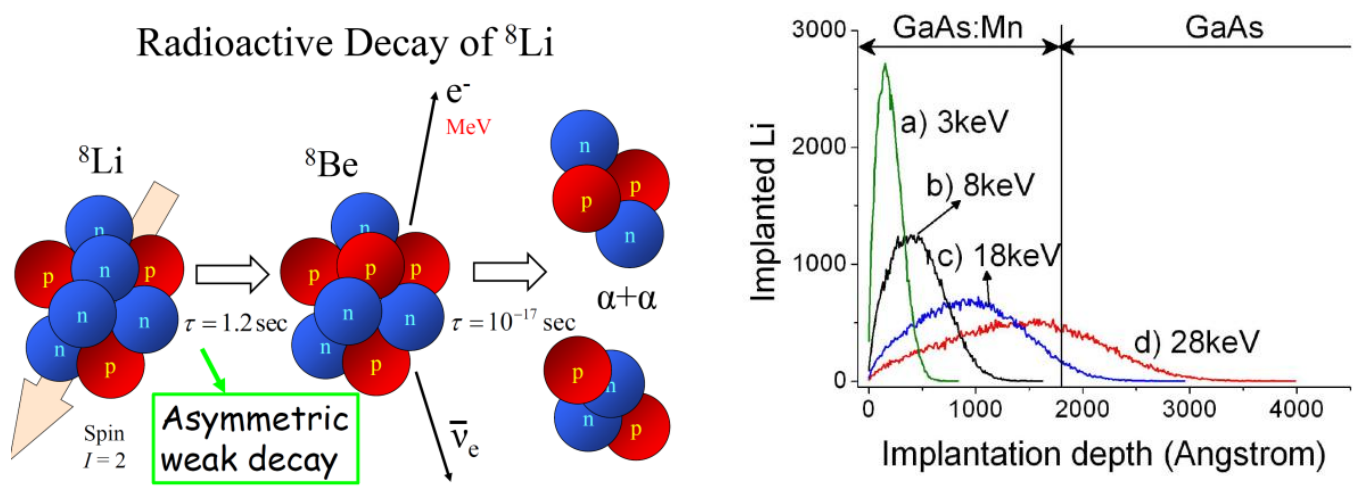

Figure 6. (left) The asymmetric weak decay of ${ }^{8} \mathrm{Li}^{+}$allows this isotope to be used analogously to $\mu^{+}$to comment on magnetic properties of materials. (right) Varying the isotope kinetic energy allows a user to choose implantation depth, and student material properties as a function of distance from a surface or interface.

\subsection{YASUTOMO UEMURA, COLUMBIA UNIVERSITY - 'FIRST-ORDER MAGNETIC QUANTUM PHASE TRANSITIONS IN MOTT INSULATORS AND UNCONVENTIONAL SUPERCONDUCTORS.'}
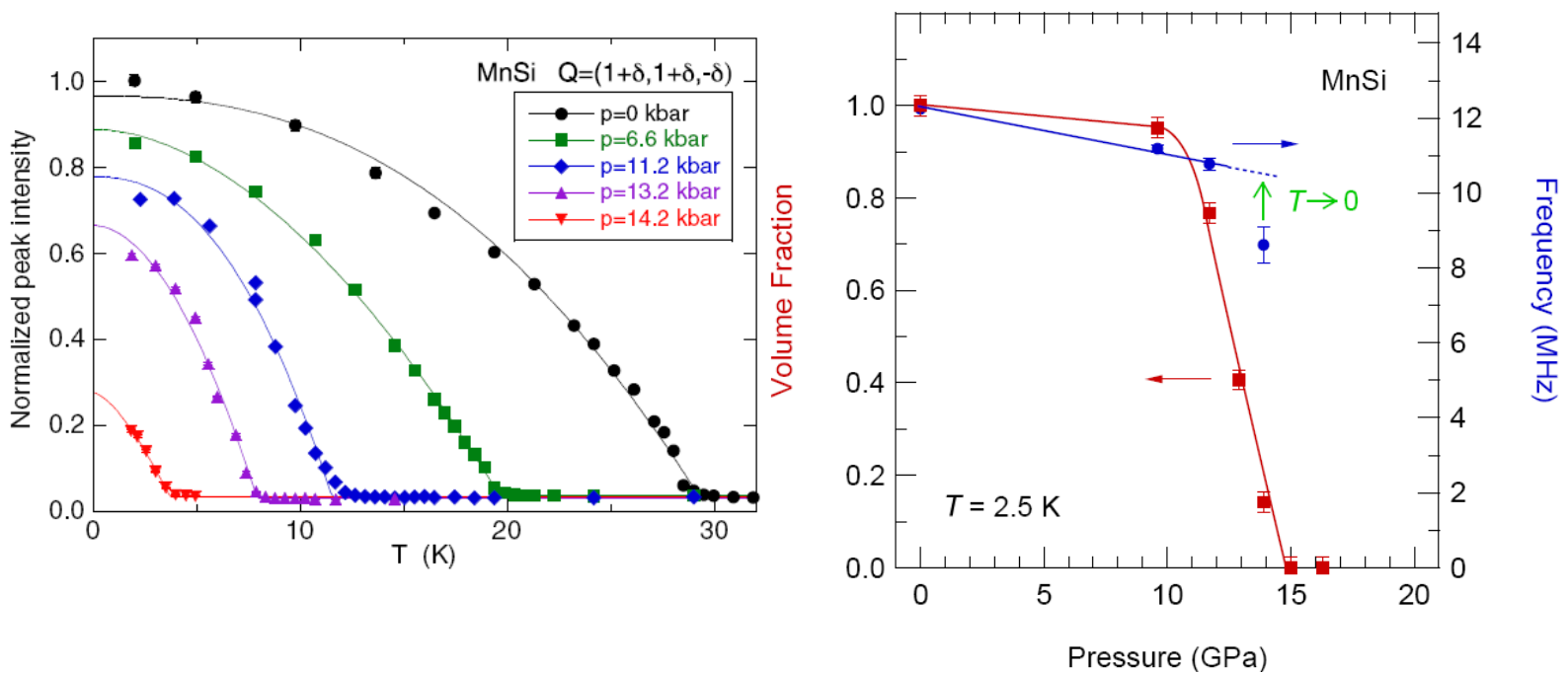

Figure 7. (left) The normalized Bragg peak intensity as a function of pressure in MnSi. (right) The pressure-dependence of the internal field (frequency) and magnetic volume fraction as measured by $\mu \mathrm{SR}$.

Dr. Uemura, Professor of Physics at Columbia University, pointed out the strong connection between the information revealed by neutron scattering and $\mu \mathrm{SR}$ experiments. In particular, he highlighted past and ongoing collaborations with neutron scatterers such as P. Dai (Rice), R.J. Birgeneau (UC Berkeley) and Ch. Pfleiderer (TU Munich) on measuring unconventional superconductors and neutron resonance phenomenology. 
An example of the complementarity of these probes, Dr. Uemura described a collaboration with Ch. Pfleiderer to measure the quantum phase transition under pressure in $\mathrm{MnBi}$ (shown in Fig. 2). While the magnetic Bragg peak measured by neutron scattering would suggest a $2^{\text {nd }}$ order transition, $\mu$ SR reveals that the since of the internal magnetic field does not change, but rather the magnetic volume fraction goes continuously to zero at the critical pressure, thereby revealing that the transition is actually $1^{\text {st }}$ order. The combination of these two techniques, a volume-averaged and a local probe, respectively, reveals more detailed information about the system than either probe could do individually.

This kind of volume-wise destruction of magnetically-ordered states shows up in many other systems as well: the Mott insulating rare-earth Nickelates $\left(\mathrm{RNiO}_{3}\right)$, vanadium oxide $\left(\mathrm{V}_{2} \mathrm{O}_{3}\right)$ and many unconventional superconductors, including the cuprates and the pnictides. These volume fraction effects suggest that fluctuations of the ordered and disordered state play a crucial role in determining the nature of the quantum phase transition. These fluctuations can be probed by by neutrons, for example through the appearance of resonance modes, and by $\mu \mathrm{SR}$, often being manifest through an increased relaxation rate. These techniques will probe fluctuations on different timescales, with neutrons measuring fluctuations on the order of $\mathrm{THz}$ while $\mu \mathrm{SR}$ measures behavior on the timescale of $\mathrm{MHz}$. In combination, these two measurements can span a much greater range of fluctuations, allowing for a more complete study of the destruction of the ordered phase.

\subsection{JEFF SONIER, SIMON FRASIER UNIVERSITY - 'SCIENCE WITH $\mu$ SR: HARD CONDENSED MATTER.'}

Dr. Sonier, Chair of the Department of Physics at Simon Frasier University, began by summarizing a proposal that was put forward in 2000 to construct a $\mu$ SR facility at the SNS, for which he was the primary organizer ${ }^{2}$. This proposal included a design that used a thin (transmission) target upstream of the SNS neutron target, which was also discussed during the breakout session (see Section 2.1). While this design had advantages and disadvantages, the proposal ultimately did not go forward because of a combined lack of support from the $\mu \mathrm{SR}$ community and from within the facility. Thus, Dr. Sonier highlighted that the successful construction of a $\mu$ SR facility being discussed in this workshop would need more engagement from the community and continued involvement of facility staff at ORNL. He particularly called on the workshop attendees to make a strong science case for the facility and to remain identifiably supportive of the project.

The presentation continued on to talk about the increasing complexity of materials and the advances in devices that use quantum mechanical phenomena. These developments in quantum materials mean that $\mu \mathrm{SR}$ must be prepared to be able to reliably measure smaller samples under extreme conditions, including high pressures, low temperature and in high magnetic fields. As an example of these kinds of developments, a new multi-crystal setup was shown (See Figure 8) that has the capacity for performing $\mu$ SR measurements on up to 6 samples simultaneously. The advances in beamline optics and detector technology would greatly increase the capabilities of a next-generation $\mu$ SR facility. 


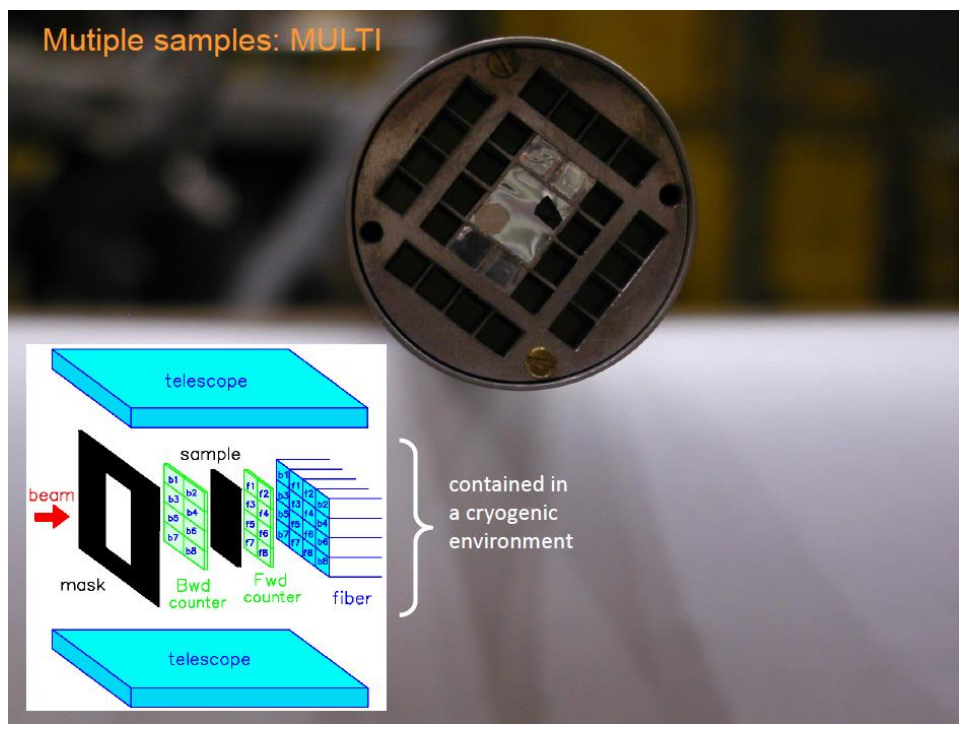

Figure 8. A picture and schematic of the MULTI sample holder for performing $\mu$ SR measurements on multiple crystals simultaneously.

It was highlighted that $\mu \mathrm{SR}$ is an important probe of these quantum materials, due to its sensitivity to local magnetism and fluctuations. As an example, the Kondo insulator $\mathrm{SmB}_{6}$ shows evidence for a topologically-protected metallic surface state ${ }^{3}$. This has been speculated to occur due to mixed $\mathrm{Sm}$ valence states that undergo Kondo hybridization in the bulk. Measurements on Fe-doped samples of $\mathrm{SmB}_{6}$ (shown in Figure 9) demonstrate that slowly-fluctuating electronic moments exist in both compounds and that the fluctuation rates are consistent with Kondo screening. The enhancement of the fluctuation rate and the smoothing of the transition with Fe doping suggests that the electronic fluctuations of the Sm moments are the key driving force behind this insulating behaviour. Furthermore, the $\mu \mathrm{SR}$ measurements are able to demonstrate that there is no phase separation into magnetic and nonmagnetic volumes at any temperature, clearly indicating that this is a bulk property of the material.
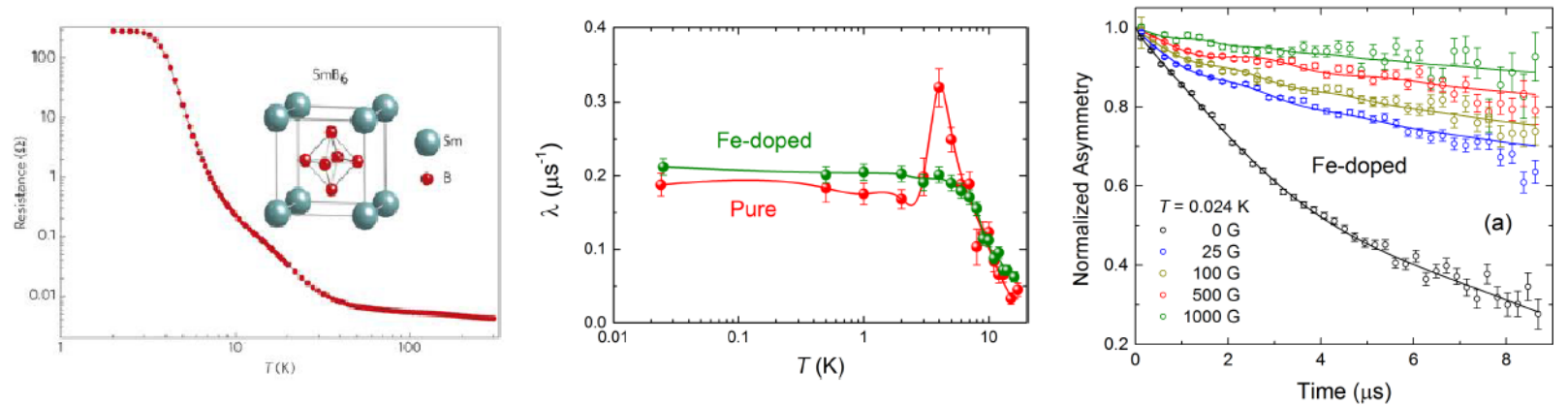

Figure 9. (left) Resistivity of $\mathrm{SmB}_{6}$ showing the onset of the bulk insulating state below $10 \mathrm{~K}$. (center) The temperaturedependent relaxation rate in pure and Fe-doped $\mathrm{SmB}_{6}$. The phase transition in the parent compound is smoothed out in the Fedoped case. (right) The $\mu$ SR spectrum as a function of field, demonstrating the existence of slow magnetic fluctuations. 


\subsection{MICHAEL GRAF, BOSTON COLLEGE - 'MAGNETISM IN $\alpha$-NAxMNO2.'}

Dr. Graf, a Professor of Physics at Boston College, described some of his recent work on $\alpha-\mathrm{NaMnO}_{2}$, and how it demonstrates the complementary nature of $\mu$ SR and neutron scattering. The complex nature of magnetism, through the coupling of various degrees of freedom, means that multiple complementary techniques are needed. In his example $\alpha-\mathrm{NaMnO}_{2}$, a material that is often studied for its applications to $\mathrm{Na}$ battery storage, there is magnetoelastic coupling. The different aspects of $\mu$ SR that have been used to study this compound mirror the different neutron scattering techniques: longitudinal field (LF)- $\mu$ SR probes spin dynamics and correlations analogous to measurements of magnetic Bragg peaks and spin waves, low- and ultra-low-energy muons can probe proximity-induced magnetism in a complementary way to neutron reflectometry, and even the development of focused muon and neutron beams for their use in measuring small samples or samples under pressure are very complementary efforts.
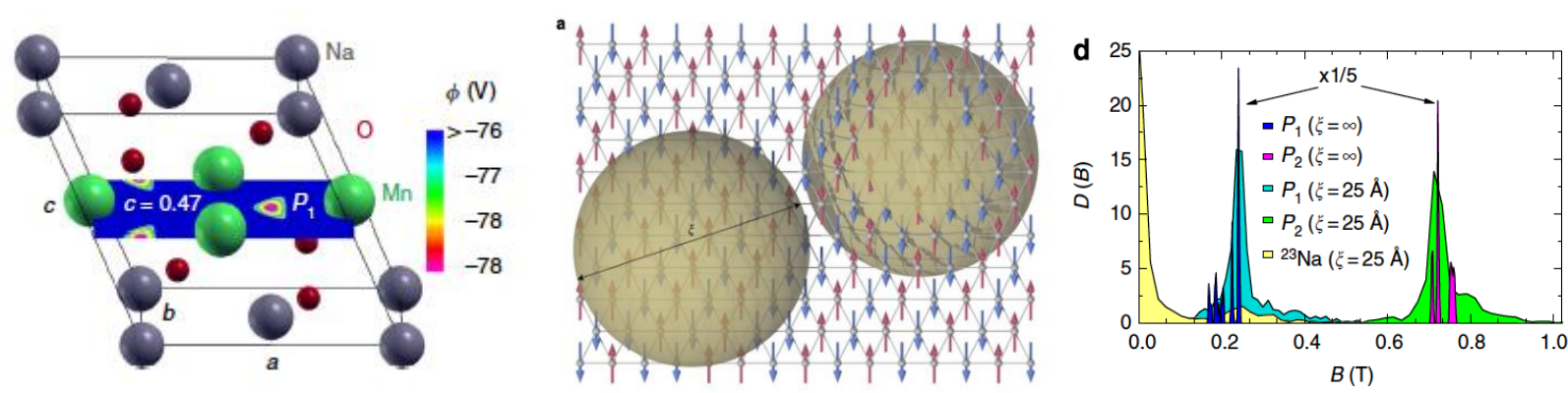

Figure 10. (left) The structure of $\alpha-\mathrm{NaMnO}_{2}$, showing the muon stopping sites. (center0 the triclinic distortions in the monoclinc bulk structure. (right) The field distribution based on the calculated stopping sites.

Another future direction raised in Dr. Graf's talk was greater integration of theory and first-principles calculations alongside $\mu \mathrm{SR}$ experiments. The community would benefit greatly from being able to routinely and quickly calculate muon stopping sites in the materials being measured, which would provide more physically significant results. This was highlighted in the data shown, where a calculation showing two muon stopping sites led to a more reasonable interpretation of the data. Within the neutron community, there already exists the capability of implementing first principles calculations alongside experiments; for example, DFT calculations are standard for experiments performed on NOMAD. This would suggest that a similar approach could be employed at a next-generation muon facility, adding physical significance to the data collected.

\subsection{GRAEME LUKE, MCMASTER UNIVERSITY - 'MUON SPIN ROTATION/ RELAXATION AS A PROBE OF UNCONVENTIONAL SUPERCONDUCTIVITY.'}

Dr. Luke, Professor and Chair of the department of Physics at McMaster University, spoke about the uses of $\mu \mathrm{SR}$ in probing unconventional superconductivity. Particularly, it was noted that the extreme sensitivity of $\mu$ SR to local magnetic fields can be used to measure the field distribution within the vortex lattice phase of a type-II superconductor. This real-space measurement of the vortex lattice can be used to measure the magnetic penetration depth, and thereby obtain the superfluid density. A $\mu$ SR measurement of the magnetic field distribution in $\mathrm{V}_{3} \mathrm{Si}$ is shown in Figure 11. The magnetic field distribution in $\mathrm{V}_{3} \mathrm{Si}$ at $\mathrm{H}=3 \mathrm{~T}$ (left) and $\mathrm{H}=5 \mathrm{~T}$ (right).. At fields just above $\mathrm{Hc}_{1}$, the field distribution is hexagonal, while closer to $\mathrm{Hc}_{2}$ the field distribution is a square lattice. This change can be seen in the $\mu \mathrm{SR}$ spectra, with the width and cut-offs of the field distribution being dependent on the magnetic penetration depth and the coherence length, respectively. 

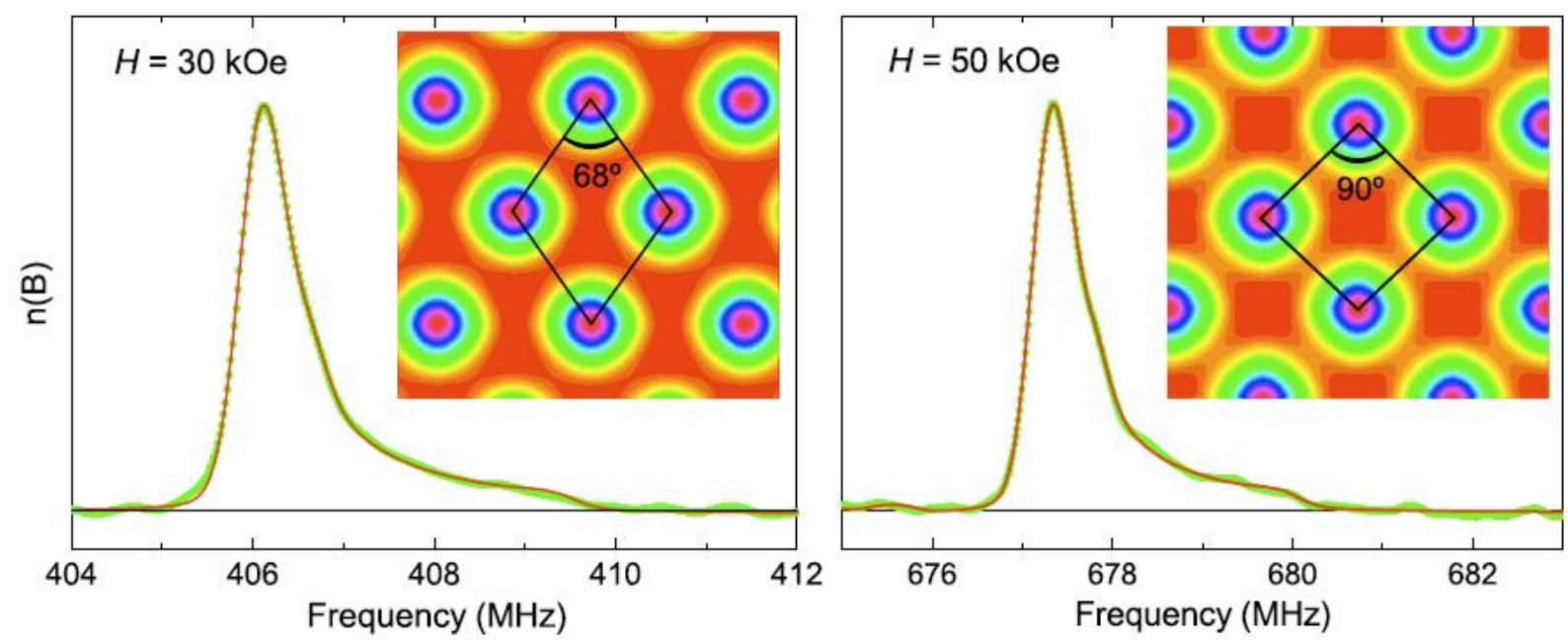

Figure 11. The magnetic field distribution in $\mathrm{V}_{3} \mathrm{Si}$ at $\mathrm{H}=3 \mathrm{~T}$ (left) and $\mathrm{H}=5 \mathrm{~T}$ (right).

This technique has been shown as a way to distinguish between different types of pairing symmetries in unconventional superconductors, including the iron pnictide superconductors and iridate superconductors. Furthermore, the ability of $\mu$ SR to measure very small magnetic fields can be used to measure time reversal symmetry breaking (TRSB) fields, in cases of $p$-wave superconductivity or other TRSB phenomena such as $\mathrm{Sr}_{2} \mathrm{RuO}_{4}$.

Both of these types of measurements have demanding experimental requirements. They often require low temperatures, applied magnetic fields and very sensitive control of the magnetic field. Thus, a future muon source would require diverse sample environments and technical support. These measurements also require high resolution, long counting times and high statistics - requirements that are strongly in line with a potential $\mu$ SR facility at the SNS.

\subsection{STEPHEN BLUNDELL, OXFORD UNIVERSITY - 'USING MUONS TO LEARN ABOUT FE-BASED SUPERCONDUCTORS AND NOVEL MAGNETS.’}

Dr. Blundell, Professor of Physics at Oxford University, spoke of his recent experiences exploring unconventional superconductors and quantum magnets with $\mu \mathrm{SR}$, placing particular emphasis on materials containing hydrogenic molecular or organic architectures. Throughout his talk, he emphasized the complementary role between $\mu \mathrm{SR}$ and other techniques employed in materials discovery efforts. He noted the continuing positive impact of $\mu \mathrm{SR}$ in the study of new superconducting families. Its relative speed and sensitivity means that the probe is ideal for constructing phase diagrams detailing the interaction of magnetic and superconducting order parameters. Because it is a real space probe, $\mu$ SR is sensitive to ordering volume fractions, and can address the question of whether phases locally co-exist or phase separate. Combining these results with x-ray diffraction and other probes, one can also include structural and electronic information and get a comprehensive picture of the physics guiding new material families. As an illustration, Dr. Blundell recounted successful efforts at Oxford in recent years to design and characterize new iron-based superconductor families containing organic spacer molecules. As a first example, data were presented from a joint $\mathrm{x}$-ray, neutron and $\mu \mathrm{SR}$ study of the newly discovered superconducting families $A_{\mathrm{x}}\left(\mathrm{NH}_{2}\right)_{\mathrm{y}}\left(\mathrm{NH}_{3}\right)_{1-\mathrm{y}} \mathrm{Fe}_{2} \mathrm{Se}_{2}$ with $A=\{\mathrm{Li}, \mathrm{Sr}$, or Rb $\}$, where $\mathrm{T}_{\mathrm{c}}$ 's can be controlled by varying either cation content or $\left(\mathrm{NH}_{2}\right) /\left(\mathrm{NH}_{3}\right)$ ratio and can reach as high as $43 \mathrm{~K}$ (compared with $\mathrm{T}_{\mathrm{c}}=9 \mathrm{~K}$ in non-intercalated $\mathrm{FeSe}$ ). Time-resolved in-situ $\mathrm{x}$-ray and neutron diffraction (on deuterated samples) were used to determine local structure and site occupancy, but $\mu$ SR played a crucial role in 
demonstrating the existence of a robust superconducting phase in the bulk of the samples, even in the presence of extensive local disorder. Muon spectra in the vortex phase further allowed for measurements of the London penetration depths, $\lambda$, and showed that these materials followed the famous Uemura relationship between $1 / \lambda^{2}$ and $T_{c}$, more commonly associated with the high- $T_{c}$ cuprate superconductors. A comparable study was shown for the material $\mathrm{Li}_{1-\mathrm{x}} \mathrm{Fe}_{\mathrm{x}}(\mathrm{OH}) \mathrm{Fe}_{1-\mathrm{y}} \mathrm{Se}$.

As a further example of complementarity, Dr. Blundell discussed his experience studying lowdimensional magnets, typically materials containing organic molecules and in which interactions between spins are strongly inhibited (but not eliminated) in one or two dimensions. The enhanced magnetic fluctuations in these materials, he pointed often obscure signatures of magnetic ordering transitions in traditional thermodynamic probes such as heat capacity and susceptibility, whereas muons oscillations remain quite clear. He pointed to several examples from his group in recent years where ordering transitions were discovered first with $\mu \mathrm{SR}$, followed by observation of subtle signatures at the ordering temperature with heat capacity. He then discussed ways in which $\mu \mathrm{SR}$ is helping test theories of lowdimensional superconductors, and the current efforts at Oxford to "engineer" dimensionality in new materials through the inclusion of hydrogen ions in existing structures.

During the latter half of the talk, Dr. Blundell discussed the series of "DFT $+\mu$ " computer codes pioneered by his group to help determine favourable muon sites inside materials of interest and quantify how the presence of the muon charge modify local structure and crystal field environments ${ }^{4}$. Muon perturbation effect, he pointed out, thought to be particularly important for systems with delicate ground states, such as frustrated spin systems, and could be associated with poorly understood reports of "persistent dynamics" in rare-earth pyrochlores or hamper efforts to detect time-reversal symmetry breaking fields in non-centrosymmetric superconductors. Specific examples were given to demonstrate how such computer simulations bolster analysis and aid in understanding $\mu \mathrm{SR}$ data, included ongoing work on the "quantum spin ice" material $\mathrm{Pr}_{2} \mathrm{Sn}_{2} \mathrm{O}_{7}$ and spin-liquid candidate $\alpha-\mathrm{RuCl}_{3}$. Determination of muon stopping sites and perturbation effects was agreed in the room to be a major ongoing concern, and there was considerable discussion of the computational and theory support desirable at any new muon source to help address this issue for new users and materials.
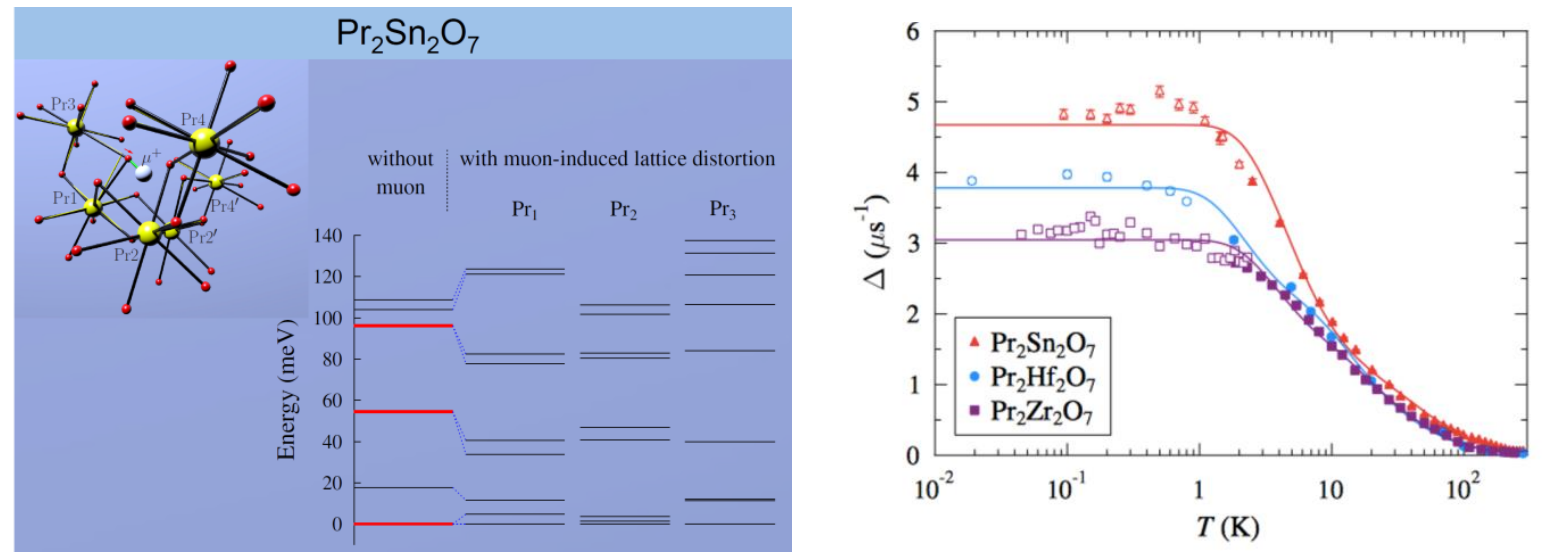

Figure 12. DFT calculations quantify the effect of a $\mu^{+}$charge on the local crystal field environment in $\operatorname{Pr}_{2} \mathrm{Sn}_{2} \mathrm{O}_{7}(\mathrm{left})$. Similar analysis allows for quantitative analysis of $\mu$ SR data from on a number of 227 pyrochore systems (right). Adapted from [4].

\subsection{IAIN MCKENZIE, TRIUMF MESON FACILITY - ' $\mu$ SR AND $\beta$-NMR OF SOFT MATTER AND CHEMICAL SYSTEMS.'}

Dr. McKenzie, Research Scientist and Outreach Coordinator for TRIUMF Meson Facility, discussed the ways that $\mu \mathrm{SR}$ and $\beta$-NMR are impacting the study of chemistry and soft matter science. When 
discussing $\mu$ SR, Dr. McKenzie pointed out that the muon acts as a light isotope of hydrogen, which can capture electrons to form a metastable "muonium" atom and form chemical bonds with host atoms in the material under study. He explained how $\mu$ SR involving such "muonated radicals" can give important information about local structure, molecular dynamics on ns- $\mu$ s timescales and reaction rate kinetics. He further gave showed how muonium can act as a near-perturbationless "spin tag", much in the same way nitroxides are used in industry, and garner information about molecular polarity and orientation within larger structures. Though brief examples were given of experiments performed in time-differential detection mode, including measurements of proton diffusion and reaction rate kinetics in liquids, the chemistry examples presented focused on measurements of hyperfine coupling constants (HCCs) of muons and host atoms, which are measured in time-integrated mode as a function of applied field. These HCCs give crucial information about electron density, structure of radicals and dynamics. He presented several examples data sets, including measurements of electron spin-relaxation in organic semiconductors, the reactivity of biradicals, the orientation of cosurfactants and micelles in bilayers, and the motion of cholesterolic molecules in a liquid crystal ${ }^{5}$.

Two interesting examples were also shown to illustrate possibilities using $\beta$-NMR as a probe. In the first, ${ }^{8} \mathrm{Li}^{+}$ions were used as an active probe, and $\beta$-NMR spectra garnered measurements of Li-diffusion rates as a function of temperature in polyethylene oxide, a common electrolyte in batteries. In the second, $\beta$-NMR was performed with ${ }^{8} \mathrm{Li}^{+}$implanted at varying depths in thin-films of polystyrene, giving evidence for enhanced fluctuations near film surfaces and an associated reduction in observed glass temperatures with decreasing film size.

Dr. McKenzie pointed out how all of these measurements depended critically on the presence of appropriate sample environment capabilities- for example the ability to apply several Tesla of field for measurements of hyperfine coupling constants- and the negative impact of poor beam stability. He emphasized, however, the central importance of total flux to overall success, especially for timeintegrated measurements. He pointed to the specific comparison of the Hifi spectrometer at ISIS and the Helios spectrometer at TRIUMF to make his point, where the order-of-magnitude greater flux in the latter allows for the measurement of more temperatures and applied field points and allows for a more complete picture of local electronic structure. The talk ended with a discussion about the size of the chemistry $\mu \mathrm{SR} / \beta$-NMR community, which was decided to make up $\sim 10-15 \%$ of the user base at European facilities. It was agreed however that, with the advent of $\beta$-NMR and low-energy $\mu \mathrm{SR}$, there was strong potential for this community to grow.
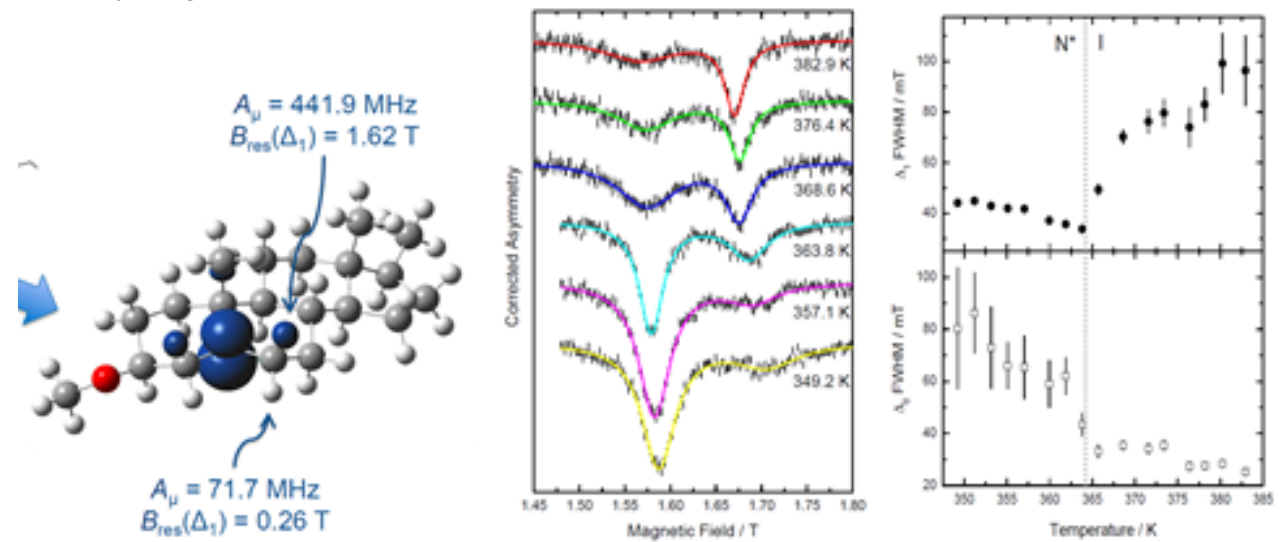

Figure 13. Example $\mu$ SR data on a particular cholesterolic liquid crystal. (left) A schematic of the cholesterol molecule, showing two muon bonding sites. (center) Time-integrated $\mu$ SR showing two distinct nuclear resonances. (right) Temperature dependence of this data reveals the presence of two distinct phases: and isotropic phase (I) at high temperatures, and a "narrowing phase" (N) where the molecules wobble around a specific axis thus reducing the width of the resonance line. Adapted from [5]. 


\subsection{ROSS CARROLL, ARKANSAS STATE UNIVERSITY - ' $\mu$ SR \& SEMICONDUCTORS.'}

Dr. Carroll, Assistant Professor of Physics at Arkansas State University, discussed the role of $\mu$ SR in exploring the physics of semiconductors. As with chemistry, muons in these systems act as hydrogen isotopes, and $\mu \mathrm{SR}$ spectra can give information about the energies, local environment, hyperfine interaction of interstitial hydrogen sites. This information is invaluable, Dr. Carroll pointed out, for locating shallow electronic defects and understanding hydrogen passivation behavior in silicongermanium alloys, which are used in photodetectors or to make high-mobility heterojunction transistors. The same information is being used to tailor electronic and optical properties in transparent conducting oxides, being used in photovoltaic or to make transparent electronics. Also mentioned was a separate line of research to explore local fields and defect behaviors in a series of magnetic semiconductors, being explored for spintronics and magneto-optics applications.

Studies of semiconductors typically employ a traditional resonance setup, where nuclear spins in a strong longitudinal field are perturbed by a transverse rf-field. With the rf-frequency fixed, longitudinal field is varying, and one searched for resonant loss of muon asymmetry. This is a time-integrated $\mu$ SR experiment, which Dr. Carroll stressed depended strongly on total muon flux. As one detailed example, Dr. Carroll presented his own work searching for shallow defect centers in silicon-germanium alloys. Using an rf-experiment as described above, his work identified two shallow muonium acceptor levels with different local environments ${ }^{6}$. The presence of both sites with different hyperfine interactions and environments, suggested that both are possible hydrogen defect centers that co-exist in this material.

Dr. Carroll spoke positively of his experiences using neutron scattering to complement his personal work with muons, and vocally supported strong ties between the two communities. He also strongly encouraged the development of multimodal capabilities wherein $\mu$ SR spectrometers are combined with, for example, the ability to perform in-situ optical pumping. He provided examples showing substantial modification of relaxation rates of muonium spins in silicon upon illuminating samples with $75 \mathrm{~W}$ light from a tungsten source. These observations, along with power and temperature dependences, were used to extract information about the activation energies and capture cross-sections of muonium sites.

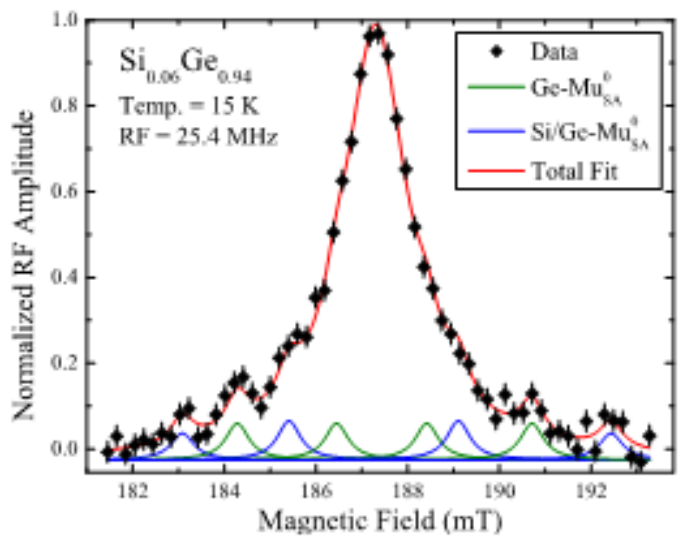

Figure 14. A recent rf-field resonant $\mu$ SR spectrum from which authors inferred the existence of two shallow muonium acceptor sites in an alloy of silicon and germanium. From [6]. 


\section{SUMMARY OF BREAKOUT SESSIONS}

\subsection{SOURCE POSSIBILITIES}

During the first breakout session, the discussion was aimed at investigating the possible source designs in more detail in order to analyze their figures of merit and which would be feasible for construction at the SNS. Three possibilities were considered: a transmission muon target placed upstream of either the FTS or STS neutron target, a solid muon target utilizing protons being directed to the beam dump of the accumulator ring, and a possibility that had materialized earlier in the day: a solid muon target utilizing a portion of the protons obtained by laser stripping the beam from the linac.

The use of a muon target placed upstream of a neutron target has been successfully employed at JPARC, ISIS and PSI. Its successful use in other facilities would mean a more straight-forward design and known source parameters, though it was noted that this method can have a substantial impact on neutron production. At PSI, for example, $\sim 2 \%$ of the proton beam is diverted towards the muon target, but the associated reduction of proton flux on the neutron target is $\sim 30 \%$, mainly due to beam scatter from the parasitic muon target upstream. A similar design idea was proposed in 2000 for a potential muon source at the SNS, utilizing the beam that would be directed to the FTS ${ }^{2}$. The associated proposal, presented during the breakout by the primary author, Prof. Jeff Sonier, included $5 \mu$ SR beamlines and utilized a 300 ns pulse with a total flux of $1 \times 10^{7} \mu^{+} / \mathrm{sec}$. Alternatively, it was suggested that the resolution could be increased by chopping the beam to get $10 \mathrm{~ns}$ pulses, which would reduce the flux to $6.6 \times 10^{4} \mu^{+} / \mathrm{sec}$. As the workshop had hit upon an alternate design, presented below, with vastly improved numbers for flux and resolution, and due to the high potential for impacting neutron production of this design, there was very little enthusiasm for pursuing this source design further.

A second source location seriously discussed would utilize the protons that would otherwise be directed to the beam dump of the accumulator ring. In contrast to the design above, this option would have no impact on neutron production at either FTS or STS. To maximize flux and simplify design, the suggested location for the muon facility in this design was near the accumulator beam stop on the west side of the ring rather than near the neutron target buildings- a suggestion that was also noted to be the preferred option for ORNL, since any additional buildings near the neutron target buildings may impact future beamline designs or modifications. Furthermore, the placement of an experimental building near the beam stop had been previously considered for the SEE facility ${ }^{1}$ and deemed feasible (see Figure 1). However, significant downsides to the use of beam dump protons were noted, including low beam power and unfavorable timing structure of the proton pulse. The beam stop absorbs $\sim 2 \%$ of the protons from the linac, with a pulse width of $1 \mathrm{~ms}$. To be useful for $\mu \mathrm{SR}$, a proton source with width comparable to the pion lifetime ( $26 \mathrm{~ns})$ is desirable. Any effort to narrow the pulse a reasonable cost would reject $99.9 \%$ of the protons. Overall, attempts to use beam dump neutrons to produce muons would be associated with a reduction relative to the FTS by a factor of $10^{2}$ due to power at the beam stop and $10^{3}$ from reducing the width of the pulse, making it uncompetitive. Again, it was noted that the third design, which used protons from the linac did not suffer from this significant intensity loss. The longer, 1 ms pulse at the beam stop would not be unfavourable for a $\beta$-NMR source, it was noted, which has a longer decay time. It was concluded that future discussions may want to consider the feasibility of using the protons directed to the beam stop for a world-leading $\beta$-NMR facility to run in parallel to existing or future materials programs.

By far the most enthusiasm of the breakout session was generated by discussion of the third source possibility, wherein it was suggested that laser stripping be used to select a proton pulse of controllable width from the linac prior to the protons entering the accumulator ring, and directing to a muon target well removed from the neutron production facility. This design presents the opportunity to take advantage of the entire amplitude of the proton current in the linac with a pulse width that is optimized for $\mu \mathrm{SR}$. If 
one used a pulse of comparable width to the pion lifetime, we estimate that one could power a $\mu$ SR facility with gain factors of $10^{1}-10^{3}$ over existing pulsed $\mu$ SR facilities (see Section A. 3). Additionally, the optimized pulse width means that this facility would have the best timing resolution of any pulsed muon source in the world. The relatively high level of control of laser-stripping technology raised the well-received prospect of "on-demand" beam timing characteristics, selecting profiles which emphasize flux or resolution as needed. This capability would be unique to ORNL. As this source option makes use of protons prior to the accumulator ring, it would have the same benefits as the beam stop design mentioned above: there would be no impact on neutron production and the muon facility would be wellseparated from the neutron target buildings. Accelerator experts at ORNL confirmed that incident power can be increased (up to $3 \%$ ) to entirely compensate for the small $(0.15 \%)$ proton current directed towards the muon target. The required laser stripping technology has been tested in limited experiments in the context for the previous SEE proposal, and shows promise for achieving the functionality necessary to this design. The principal investigators of this technology are based at ORNL, and so we will perform the necessary calculations with them to ensure that this design can be successfully achieved.

Finally, it was noted that by utilizing ideas that had been generated during the design of the SEE facility, such as the laser stripping and building location, it may be an option to construct a single facility for both purposes. Any future work on a proposed $\mu$ SR facility should explore the possibility of a joint SEE test facility, or leave open the possibility of a future expansion for that purpose.

\subsection{TARGETS AND BEAMLINES}

The second breakout session was aimed at discussing the most effective target and beamline configurations for the proposed facility. Discussions touched on the merits of having one, two or more than two muon targets; whether it was feasible to add decay muon and/or low-energy muon beamlines in addition to conventional surface muon beamlines; and how to plan for future expansion of the facility's capabilities.

Focusing on the third source design above with a dedicated proton beam for muon experiments, discussion centered on dedicated target designs, with the stated aim of maximal use of available protons. Discussion centered on three main target designs possibilities: a single, solid target; a smaller transmission target in front of a second, solid target; or a long, solid target that aims to stop the protons over a range of distances. The first design, utilizing a single, solid target, is the most straightforward and well understood. Any instruments arranged around this target will receive the same flux of surface muons, also simplifying the design and versatility of the facility. The second design would feature two targets: the first would be aimed at stopping a fraction of the muons, with the rest continuing downstream into a second, stopping target. The benefits of this design are that it would allow a greater production of surface muons, since each target would be smaller than the previous case, increasing surface to volume ratio. This concept would also accommodate more room in the experiment hall for more complex instrument designs. It may also allow the upstream target to operate independently of the downstream target. However, protons passing through the first target will undergo moderate scattering, reducing the flux on the second target by $\sim 20-30 \%$. It was noted that this design would also require more shielding and beamline components, with associated increase in cost. The third target concept considered was a long extended target, wherein surface muons were produced at the sides of the target over a range of distances. This novel design would enable an increased production of surface muons relative to the single target design, while reducing proton scattering that occurs in the second design. However, the production of decay muons in this design would be diminished, as they are produced in the forward (downstream) direction in the target. This design concept may also prove difficult to steer the muons into beamlines, since they are created over a comparatively long length. While no clear consensus emerged from the discussions of target designs, it was felt that each of these concepts had clear merits that warranted further 
study. A fourth design presented below, where a kicker element alternately directs protons towards separate muon targets, was suggested rather late in the discussion and not fully contemplated.

It was realized that the high flux offered by the favored source design would be capable of supporting beams of surface muons, decay muons, as well as low energy muons. While the surface muons being the most conventional type of $\mu$ SR beam, being used for most magnetism, superconductivity and chemistry experiments, the decay and low energy muon beams would bring substantially more capability to a $\mu$ SR facility at the SNS and help expand the community. Decay muon beamlines can provide a means to enhance the stopping depths, allowing measurements of samples in pressure cells or other containment vessels, as well as larger, non-uniform components such as mechanical or electronic devices. These capabilities are an area of strong growth within the neutron program at ORNL, and one or more decay muon beamlines would bring strongly complementary measurements to this in-house research program. Similarly, the inclusion of low-energy muon beams would also form strong connections to ongoing research areas at ORNL. Low-energy beams have a reduced stopping depth, enabling depth-resolved measurements in thin films, devices and nanostructures, as well as allowing for the measurement of surface properties. It was noted that enabling a beamline to be switched between conventional surface muons and low-energy muons would allow for single experiments that can simultaneously measure bulk and surface properties. Decay and low-energy muon beams are strongly desired by the community; of the 17 current $\mu$ SR beamlines, 7 are decay muon beamlines and only 1 is a low-energy muon beamline (note: only 5 of the decay muon beamlines are currently operational; see Appendix C).

Based on the calculations of the muon flux that could be generated at the SNS (see Section A. 2.), it was felt that 4 surface muon beamlines, as well as 1-2 decay muon beamlines could easily be feasible. Furthermore, it was deemed scientifically and technically valuable to include the capacity for 1-2 low energy muon beamlines, though specific intensity calculations would need to be performed to assess feasibility. A world-leading $\beta$-NMR source utilizing beam dump protons was also deemed viable, and could be operated in parallel to the muon source from laser-stripped protons, while sharing building infrastructure. Such an ion source could be constructed at the same time as the muon facility for minimal cost, or as a later expansion. More generally, it was felt that the contingency for future expansion should be considered when creating a full design of the facility. As one possibility, it was noted that it may be possible to use an electrostatic kicker to direct the proton pulse alternately to two muon targets. This would require the laser stripping to operate with double the duty cycle, taking a proton pulse every $10 \mu \mathrm{s}$, such that each target would then receive a pulse every $20 \mu \mathrm{s}$. In this way, the number of muons and beamlines at the facility could be doubled if such an expansion was deemed a productive investment. It would also allow for more capabilities and unforeseen future technologies to be implemented in a woldclass $\mu$ SR facility.

\subsection{SCIENCE DRIVERS}

The ideas presented in the talks stimulated much discussion on the scientific impact of a nextgeneration muon source at the SNS during the final breakout session. It was felt that the muon source that was outlined in the previous breakout sessions would be able to address new and innovative science questions, owing to the substantial improvement in flux and resolution that this source would allow, as well as closer integration with computation, detector and sample-environment expertise at ORNL.

In condensed matter physics, the timing structure of pulsed sources create more useful muons per second relative than continuous sources and offer the opportunity to measure to longer time spectra, making accessible the detection of smaller moments and slower relaxation phenomena. However, the maximum moments and faster relaxations, and well as magnitude of applied fields, are tightly constrained by the timing resolution of the pulsed $\mu$ SR experiments, tied intimately to intrinsic width of the muon 
pulse. A muon source at the SNS would have better flux and resolution than any current source of muons, expanding the range of phenomena available to be measured and while pushing increasing the flexibility of the technique. In particular, it increases the timescale of fluctuations that can be detected, which was noted as being strongly complimentary to inelastic neutron scattering. It was also discussed that the determination of muon stopping sites and perturbative effects, an area of intense current focus in the field, would strongly benefit from closer integration with computational resources and expertise at ORNL. Based on the current state-of-the-art at neutron beamlines at ORNL, it was suggested and greatly encouraged that any new source include specialized software, perhaps on the beamline, which would greatly increase the information that can be determined from $\mu$ SR experiments. The complementarity of $\mu$ SR to not only neutrons but also traditional thermodynamic probes was noted, and the technique was demonstrated to be well-suited to the study of phase transitions. This is due to $\mu$ SR being sensitive to local fluctuations, real-space phase separation and dynamics on a microsecond timescale, which is in turn dependent on the resolution and flux of the source. Thus, a next-generation source at the SNS would push highly sensitive measurements of magnetic phase transitions, already well-developed area of research, into new territory.

Significant discussion was dedicated to the size of the $\mu$ SR community, expansion opportunities, and the positive impact a new source in the United States. One relatively under-developed field, it was noted, is $\mu$ SR studies of biological systems. Such measurements been historically limited by the lack of muon site information, multiple relaxation mechanisms and effects due to muon hopping effects. It is thought that computational support and increased flux might make a positive impact in this regard. The potential to include low-energy capable beamlines would also open significant avenues of research in thin films, devices and nanostructures $-\mu$ SR research community that has been rapidly expanding since the advent of low-energy beams at PSI. With the expansion of worldwide research devoted to these areas using all available techniques, along with recent interest in surfaces and interface effects, low-energy $\mu$ SR and $\beta$ NMR are expected to be significant growth areas. The workshop participants also noted that the $\mu$ SR community has benefitted from several close collaborations with industry in the past few years. In particular, Toyota has used $\mu$ SR to study Li diffusion in battery compounds, while Cisco has performed work studying muon irradiation in electronic components. Industrial partnerships are thought to be likely to grow in frequency, with various industry partners able to benefit from studying muon irradiation or hydrogen chemistry through the use of $\mu$ SR. Such measurements, it is noted, would be greatly enhanced by the higher flux that would be present at the proposed SNS muon source. Furthermore, by incorporating new developments in focusing optics, it has been proposed that experiments can be performed while scanning the muon beam over different regions of the sample. As a local probe with a small penetration depth, this would provide information on the millimetre length scale across different portions of the sample. Similarly, focusing optics would enable the measurement of milligram-sized samples, or samples in pressure cells. The increased muon flux further enhances this capability, allowing measurements to be performed on samples that are out of the range of current technology.

Through the breakout session, and throughout the meeting, it was repeatedly and strongly expressed by the participants that the co-location of a muon source with the existing and planned neutron sources at ORNL would be greatly beneficial for both techniques. The scientific complementarity of the two experiments has been illustrated above, but it was also highlighted by many that the user communities of the two techniques also strongly overlap. At two of the European muon sources that are co-located with a neutron source, ISIS and PSI, 10-15\% of the neutron users are also users of the muon source. This is strongly aided by joint user programs in the two facilities and reciprocal beamtime agreements. With upwards of 680 unique users at the SNS and HFIR each year, the same rate of users for an SNS muon source would more than double the number of North American muon source users, without accounting for the new capabilities made possible by a next-generation muon source design as is being discussed.. It was also suggested that partnership with the strong programs available for students at ORNL, such as the 
annual Neutron \& X-ray Summer School, would provide another opportunity to increase awareness of the technique and grow the $\mu$ SR community in the United States. 


\section{CONCLUSIONS}

As laid out in detail above, the conclusions from this workshop were overwhelmingly positive with regard to the state of the $\mu$ SR community and possibilities for a source at the SNS. In addition to presentations that reinforced the scientific worth of the technique, several strong cases were made that demonstrate the ongoing development of $\mu$ SR around the world: increasing user numbers at existing $\mu$ SR facilities, increasing collaboration with neutron scatterers and other researchers in materials science, the opening of the technique to new communities, and several recent discussions for expansion possibilities for $\mu$ SR facilities. These developments would only be further accelerated by the construction of a USbased $\mu$ SR source.

Indeed, the most exciting conclusion from the meeting was the entirely unanticipated development of a novel source design at the SNS, which would use existing laser stripping technology (developed at ORNL) to create a muon source with world-leading numbers for resolution and flux, and a unique control of beam characteristics. The design was such that the muon source, if constructed, would have negligible negative impact on the existing or future neutron programs, but a large positive impact on the overall user program, resulting from the co-location of two powerful probes of matter. The meeting ended with strong encouragement from all involved that this source design be developed, to both assess feasibility and further explore possible impacts such a world-leading facility could have on the materials community in the US and globally. 


\section{REFERENCES}

${ }^{1}$ B.W. Riemer and F.X. Gallmeier. Definition of Capabilities Needed for a Single Event Effects Test Facility. Oak Ridge National Laboratory. Internal Document \#ORNL/TM-2014/553. (2014).

${ }^{2}$ J.E. Sonier et al. A Next Generation U.S. Muon Facility. (2000).

${ }^{3}$ D.J. Kim, J. Xia and Z. Fisk. Nat. Mat. 13, 466 (2014).

${ }^{4}$ F.R. Foronda, F. Lang, J.S. Möller, T. Lancaster, A.T. Boothroyd, F.L. Pratt, S R. Giblin, D. Prabhakaran, and S.J. Blundell. Phys. Rev. Lett. 114, 017602 (2015).

${ }^{5}$ I. McKenzie, R. Scheuermann, K. Sedlak and A. Stoykov. J. Phys. Chem. B 115, 9360 (2011).

${ }^{6}$ B.R. Carroll. R.L. Lichti, P.W. Mengyan, B.B. Baker, Y.G. Celebi, P.J.C. King, K.H. Chow, and I. Yonenaga. Appl. Phys. Lett. 105, 122101 (2014). 
APPENDIX A. SOURCE CALCULATION 


\section{A. 1. SUMMARY}

Following the discussions reported in Section 2, it became clear that the muon source design that was most feasible and best for addressing the scientific challenges was one that utilized the laser stripping for directing protons to a dedicated muon target. Compared to a transmission target that was placed upstream of the FTS or STS neutron target, a dedicated muon target would have no impact on neutron production or the layout of the FTS or STS target buildings. Furthermore, while a muon target that utilized the protons that are being directed to the primary and/or secondary beam stop would also have no impact on neutron proton, but has a pulse timing structure that is not optimal for muon production.

The ability of the laser stripping technique to produce an optimal pulse structure for powering a muon source, combined with the high power of the SNS accelerator has the potential to facilitate the world's highest flux and best resolution pulsed muon source. The following sections outline the calculation of the muon flux and resolution (Section 30A. 2) of this potential facility, and its comparison to the world's 4 existing muon facilities (Section A. 3). The workshop was not tasked with preparing a full design, so the values given below are approximations based on reasonable assumptions about a future facility design. However, it was the conclusion of all of the workshop participants that these were realistic parameters, and that this facility would vastly exceed the capabilities of the existing muon sources around the world.

\section{A. 2. DETAILED CALCULATION}

After the completion of the PPU project, the accelerator will be operating with the following specifications:

\begin{tabular}{|l|l|}
\hline Accelerator Power & $2.47 \mathrm{MW}$ \\
\hline Proton Energy & $1.3 \mathrm{GeV}$ \\
\hline Current & $1.5 \mathrm{~mA}$ \\
\hline Protons per Pulse & $2.5 \times 10^{14}$ \\
\hline Pulse Frequency & $60 \mathrm{~Hz}$ \\
\hline Operating Days per year & $\mathrm{TBD}($ Currently $\sim 200)$ \\
\hline
\end{tabular}

Table 1. The operational parameters of the SNS accelerator after the PPU project.

Then the protons per second coming from the Linac can be found by:

$$
\begin{aligned}
\text { Protons per second } & =(\text { Protons per Pulse }) \times(\text { Pulse Frequency }) \\
\text { (accelerator) } & =2.5 \times 10^{14} \times 60 \mathrm{~Hz} \\
& =1.5 \times 10^{16} \mathrm{p}^{+} / \mathrm{sec}
\end{aligned}
$$

The optimal beam for a muon source is a pulse width that is short compared to the muon lifetime (2.2 $\mu \mathrm{s})$ and has pulse separation that is long compared to the muon lifetime. However, any proton pulse will be broadened by the pion lifetime ( $26 \mathrm{~ns}$ ) during the proton-pion-muon conversion in the target. Thus, the optimal proton pulse for this muon source will be $30 \mathrm{~ns}$ width, with $20 \mu$ s between pulses. This was decided through discussion to be the optimal balance of resolution and flux.

$$
\begin{aligned}
\text { Duty cycle } & =(\text { On time }) /(\text { Off time }) \\
& =30 \mathrm{~ns} / 19.97 \mu \mathrm{s} \\
& =3 \times 10^{-8} \mathrm{~s} / 1.997 \times 10^{-6} \mathrm{~s} \\
& =0.0015 \\
& =0.15 \%
\end{aligned}
$$


The number of protons per second in the muon beam is:

$$
\begin{aligned}
\text { Protons per second } & =(1) \times(2) \\
\text { for muon source) } & =1.5 \times 10^{16} \times 0.0015 \\
& =2.25 \times 10^{13} \mathrm{p}^{+} / \mathrm{sec}
\end{aligned}
$$

The laser stripping technique has been demonstrated ${ }^{7}$ to be $\sim 90 \%$ efficient, however at this duty cycle, the most feasible design would utilize a $4 \mathrm{MW}$ laser in a 12 -reflection optical cavity ${ }^{8}$, which would reduce the efficiency to $\sim 70 \%$ and broaden the time-width of the laser pulse (See Figure 15).
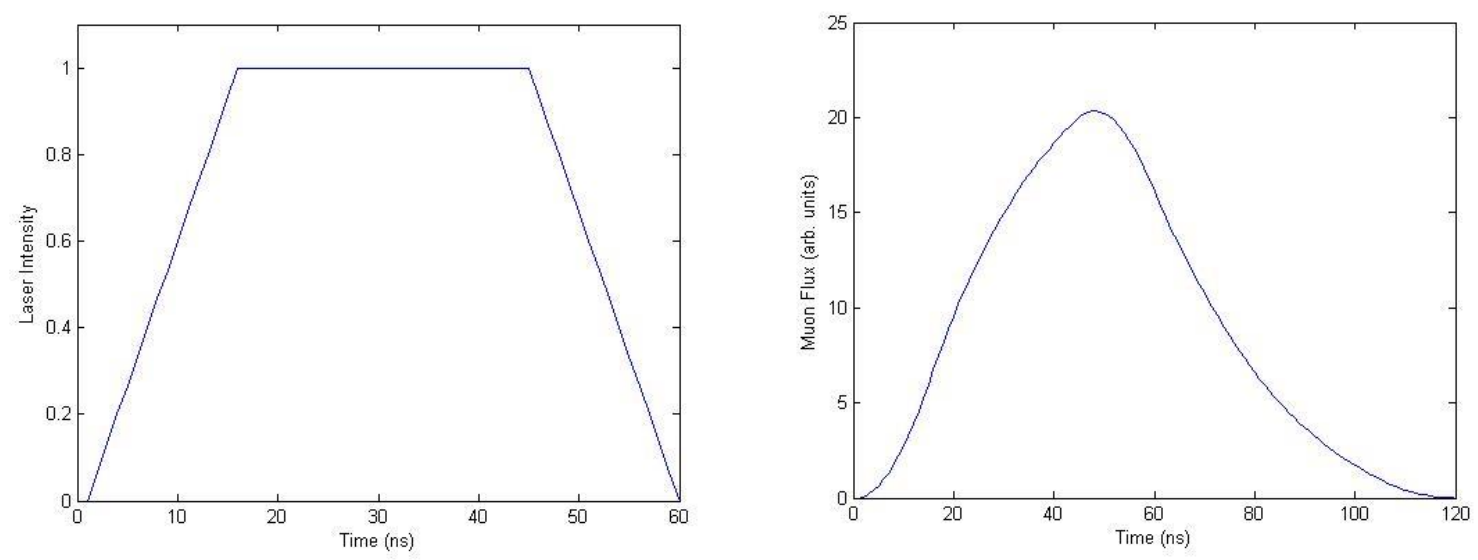

Figure 15. (left) The intensity of the proposed laser pulse as a function of time, with $30 \mathrm{~ns}$ at peak intensity. (right) The resulting time structure of the muon pulse, resulting from the convolution of the laser pulse shape with the pion lifetime.

The overall intensity of the laser pulse (left panel of Figure 15) is 50\% larger than the optimal square pulse of 30ns duration, due to the ramping of the laser. Additionally, the full width at half maximum of the resulting muon pulse (right panel of Figure 15) is $50 \mathrm{~ns}$, containing $77.94 \%$ of the total muons produced. Accounting for both of these factors, as well as the efficiency of the laser stripping, the number of protons per second that will be diverted to the muon target is:

$$
\begin{aligned}
\text { Protons per second } & =(3) \times(\text { Intensity factor }) \times(\text { Efficiency }) \\
\text { (to muon target) } & =2.25 \times 10^{13} \times 1.50 \times 0.70 \\
& =2.363 \times 10^{13} \mathrm{p}^{+} / \mathrm{sec}
\end{aligned}
$$

The estimate of the proton-pion-muon conversion rate for a solid (thick, stopping) target is difficult to calculate, since all current targets are transmitting targets, placed upstream of other targets (muon and/or neutron targets). A Monte Carlo simulation was performed using the specification of the $\mathrm{T} 1$ target at TRIUMF assuming $1.3 \mathrm{GeV}$ incident protons, which gave a conversion factor of $3.6 \times 10^{-7} \mu^{+} / \mathrm{p}^{+9}$, where we are only counting muons produced from pion decay at rest (surface muons). It is very likely that a target design that aims to stop all of the incident protons would produce a higher muon flux, but this is hard to estimate without more in-depth analysis of potential target designs. Using the factor from the simulation, this would give the number of muons produced, per second, as:

$$
\begin{aligned}
\text { Muons per second } & =(4) \times(\text { Conversion rate) } \\
\text { (from muon target) } & =2.363 \times 10^{13} \times 3.6 \times 10^{-7} \\
& =8.505 \times 10^{6} \mu^{+} / \mathrm{sec}
\end{aligned}
$$


This is the total number of muons produced, which will be distributed evenly over $4 \pi$ steradians of solid angle. If we assume that instruments can cover $\sim \pi$ steradians of solid angle, the number of muons going to the beamlines will be:

$$
\begin{aligned}
\text { Muons per second } & =(5) \times(\text { Angular coverage }) / 4 \pi \\
\text { (to instruments) } & =8.505 \times 10^{6} \times \pi / 4 \pi \\
& =2.126 \times 10^{6} \mu^{+} / \mathrm{sec}
\end{aligned}
$$

This angular coverage should be achievable with the use of bending magnets, since the muons are positively charged, allowing a large fraction of the produced muons to be directed to the beamlines.

If this flux was used to support 4 beamlines, each muon beamline receiving $1 / 4$ of the muons produced, the flux at each beamline would be:

$$
\begin{aligned}
\text { Muons per second } & =(6) / 4 \\
\text { (per instrument) } & =2.126 \times 10^{6} / 4 \\
& =5.316 \times 10^{5} \mu^{+} / \mathrm{sec}
\end{aligned}
$$

The width of the muon pulse would be a convolution of the width of the proton beam and the pion lifetime (26 ns). For the shape shown in Figure 15, the width is:

$$
\begin{aligned}
\text { Muon pulse width } & =\left[(\text { Proton pulse width })^{2}+(\text { Pion Lifetime })^{2}\right]^{1 / 2} \\
& =\left[\left(4.25 \times 10^{-8}\right)^{2}+\left(2.6 \times 10^{-8}\right)^{2}\right]^{1 / 2} \\
& =49.8 \mathrm{~ns}
\end{aligned}
$$

This is the shorter than any other muon source and offers the distinct advantage that the laser pulse length would be tunable. So while this calculation offers the flux characteristics for one given resolution, the entire facility could offer variable resolution depending on the parameters of the experiment.

This is an initial calculation, but would suggest that this source design would lead to a pulsed muon beam with $50 \mathrm{~ns}$ width and $8.5 \times 10^{6} \mu^{+} / \mathrm{sec}$ intensity, capable of supporting 4 muon beamlines. It was also pointed out during the workshop discussion that the proton-pion-muon conversion in the target would produce approximately 10 times more decay muons $\left(\sim 8 \times 10^{7} \mu^{+} / \mathrm{sec}\right)$, ranging in momentum from 4.119 $\mathrm{MeV}$ to $1.3 \mathrm{GeV}$. These could be used to support one or more decay muon beamlines for measuring thick samples or samples in pressure cells. Employed as a low-energy muon beamline, the flux would be reduced by a factor of $10^{4}-10^{5}$, which would suggest that the muon flux for a low-energy muon beam would be $\sim 4 \times 10^{3} \mu^{+} / \mathrm{sec}$. This suggests that while the resolution would be limited by the pulse width, the muon flux would be high enough to perform experiments using low-energy muons, being comparable to the low energy muon flux at the only operational low energy muon beamline. This would open up new capabilities for measuring thin films and nano-structured materials.

\section{A. 3. COMPARISON TO EXISTING SOURCES}

There are four existing muon sources around the world, of which two are continuous sources and two are pulsed sources (see Table 2). In terms of source characteristics, it makes sense to discuss pulsed and continuous sources separately. This is because while pulsed sources have much higher flux and can observe longer signal times, continuous sources have very little background from their lack of pulse width. This makes pulsed sources more amenable to measuring slowly relaxing phenomena and smaller magnetic fields, while continuous sources are better suited to faster fluctuations and small volume fractions. 


\begin{tabular}{|l|l|l|}
\hline Facility & Location & Type of Source \\
\hline $\begin{array}{l}\text { ISIS } \\
\text { (Rutherford Appleton Laboratory) }\end{array}$ & Oxford, United Kingdom & Pulsed \\
\hline $\begin{array}{l}\text { J-PARC } \\
\text { (Japan Proton Acceleratory Research }\end{array}$ & Tokai, Japan & \\
Complex) & & Pulsed \\
\hline $\begin{array}{l}\text { PSI } \\
\text { (Paul Scherrer Institute) }\end{array}$ & Villigen, Switzerland & Continuous \\
\hline $\begin{array}{l}\text { TRIUMF } \\
\text { (TRIUMF Meson Facility) }\end{array}$ & Vancouver, Canada & Continuous \\
\hline
\end{tabular}

Table 2 . Name, location and facility type of the 4 muon sources around the world.

The flux and resolution (pulse width) are the two most important characteristics for a pulsed muon source. The source design outlined in the previous section has a pulse width of $50 \mathrm{~ns}$, which gives better resolution than the source at ISIS (70 ns) and equal to that at J-PARC (50 ns). Furthermore, the high power of the SNS accelerator, and the ability to strip $\sim 70 \%$ of the protons with the laser means that a muon source at the SNS would have a higher flux $\left(8.505 \times 10^{6} \mu^{+} / \mathrm{sec}\right)$ to either ISIS $\left(1.5 \times 10^{6} \mu^{+} / \mathrm{sec}\right)$ and J-PARC $\left(1.8 \times 10^{6} \mu^{+} / \mathrm{sec}\right)$. The calculation of the flux in Section A. 2 was a first approximation, and may be increased when designed with a more efficient target. This may result in the proposed facility having an even higher flux than is currently available anywhere in the world. 
APPENDIX B. PARTICIPANTS, PRESENTATIONS AND AGENDA 


\section{APPENDIX B.1 PARTICIPANTS}

Table 3. Table of workshop participants.

\begin{tabular}{|l|l|l|}
\hline Name & Institution & Email address \\
\hline Adam Aczel & ORNL & aczelaa @ ornl.gov \\
\hline Stephen Blundell & Oxford University & stephen.blundell @ physics.ox.ac.uk \\
\hline Ross Carroll & Arkansas State University & bcarroll@ astate.edu \\
\hline John Galambos & ORNL & galambosjd@ornl.gov \\
\hline Michael Graf & Boston College & michael.graf.bc.edu \\
\hline Adrian Hillier & ISIS, Rutherford-Appleton Laboratories & adrian.hillier@ stfc.ac.uk \\
\hline Syd Kreitzman & TRIUMF & syd @triumf.ca \\
\hline Graeme Luke & McMaster University & luke@ momaster.ca \\
\hline Mark Lumsden & ORNL & lumsdenmd@ ornl.gov \\
\hline Gregory MacDougall & University of Illinois, Urbana-Champaign & gmacdoug@illinois.edu \\
\hline Andrew MacFarlane & University of British Columbia & wam@ chem.ubc.ca \\
\hline Doug MacLaughlin & University of California, Riverside & macl@ citrus.ucr.edu \\
\hline Iain McKenzie & TRIUMF & iain.mckenzie@ triumf.ca \\
\hline Elvezio Morenzoni & Paul Scherrer Institute & elvezio.morenzoni @ psi.ch \\
\hline Stephen Nagler & ORNL & naglerse@ ornl.gov \\
\hline Mike Plum & ORNL & plumma @ ornl.gov \\
\hline Robert Plunkett & Fermilab & plunk@ fnal.gov \\
\hline Bernie Riemer & ORNL & riemerbw@ ornl.gov \\
\hline Koichiro Shimomura & Japan Proton Accelerator Research Complex & koichiro.shimomura@ kek.jp \\
\hline Jeff Sonier & Simon Frasier University & jsonier@ sfu.ca \\
\hline Alan Tennant & ORNL & tennantda@ ornl.gov \\
\hline Yasutomo Uemura & Columbia University & tomo@lorentz.phys.columbia.edu \\
\hline Travis Williams & ORNL & williamstj@ornl.gov \\
\hline & & \\
\hline
\end{tabular}




\section{APPENDIX B.2 PRESENTATIONS}

Table 4. Table of workshop presentations.

\begin{tabular}{|c|c|c|}
\hline Name & Institution & Title \\
\hline Stephen Blundell & Oxford University & $\begin{array}{l}\text { Using Muons to Learn about Fe-based } \\
\text { Superconductors and Novel Magnets }\end{array}$ \\
\hline Ross Carroll & Arkansas State University & $\mu$ SR \& Semiconductors \\
\hline John Galambos & ORNL & $\begin{array}{l}\text { SNS: The Second Target Station (STS) } \\
\text { Upgrade Project Design Status }\end{array}$ \\
\hline Michael Graf & Boston College & Magnetism in $\alpha$-NaxMnO2 \\
\hline Adrian Hillier & $\begin{array}{l}\text { ISIS, Rutherford-Appleton } \\
\text { Laboratories }\end{array}$ & Muons at ISIS \\
\hline Syd Kreitzman & TRIUMF & $\begin{array}{l}\text { Overview of the TRIUMF Centre for } \\
\text { Molecular and Materials Science }\end{array}$ \\
\hline Graeme Luke & McMaster University & $\begin{array}{l}\text { Muon Spin Rotation/Relaxation as a Probe of } \\
\text { Unconventional Superconductivity }\end{array}$ \\
\hline Andrew MacFarlane & University of British Columbia & $\begin{array}{l}\beta \text {-detected NMR at TRIUMF: Status and } \\
\text { Progress }\end{array}$ \\
\hline Iain McKenzie & TRIUMF & $\begin{array}{l}\mu \mathrm{SR} \text { and } \beta \text {-NMR of Soft Matter and } \\
\text { Chemical Systems }\end{array}$ \\
\hline Elvezio Morenzoni & Paul Scherrer Institute & $\begin{array}{l}\text { Muons for Solid State Research at the Paul } \\
\text { Scherrer Institute }\end{array}$ \\
\hline Stephen Nagler & ORNL & \\
\hline Mike Plum & ORNL & SNS Muon Target Possibilities \\
\hline Koichiro Shimomura & $\begin{array}{l}\text { Japan Proton Accelerator } \\
\text { Research Complex }\end{array}$ & Status of J-PARC Muon Science Experiment \\
\hline Jeff Sonier & Simon Frasier University & Science with $\mu$ SR: Hard Condensed Matter \\
\hline Alan Tennant & ORNL & Neutron Sciences at ORNL \\
\hline Yasutomo Uemura & Columbia University & $\begin{array}{l}\text { First-Order Magnetic Quantum Phase } \\
\text { Transitions in Mott Insulators and } \\
\text { Unconventional Superconductors }\end{array}$ \\
\hline
\end{tabular}




\section{Future Muon Source Possibilities at the SNS Workshop September 1-2, 2016}

Thursday, September 1, 2016

Clinch River Cabin

\begin{tabular}{|c|c|c|}
\hline Time & Event & Speaker \\
\hline 9:00-9:15 AM & Opening Remarks and Workshop Goals & Travis Williams \\
\hline $9: 15-9: 45$ am & Neutron Science at ORNL & Alan Tennant \\
\hline $9: 45-10: 15$ am & $\begin{array}{l}\text { SNS: The Second Target Station (STS) Upgrade } \\
\text { Project Design Status }\end{array}$ & $\begin{array}{l}\text { John Galambos / Mike } \\
\text { Plum }\end{array}$ \\
\hline 10:15-11:00 am & Break & \\
\hline $11: 00-11: 30 \mathrm{am}$ & Muons at ISIS & Adrian Hillier \\
\hline $11: 30-12: 00 \mathrm{pm}$ & Status of J-PARC Muon Science Experiment & Koichiro Shimomura \\
\hline $12: 00-1: 00 \mathrm{pm}$ & Lunch & \\
\hline $1: 00-1: 30 \mathrm{pm}$ & $\begin{array}{l}\text { Muons for Solid State Research at the Paul Scherrer } \\
\text { Institute }\end{array}$ & Elvezio Morenzoni \\
\hline $1: 30-2: 00 \mathrm{pm}$ & $\begin{array}{l}\text { Overview of the TRIUMF Centre for Molecular and } \\
\text { Materials Science }\end{array}$ & Syd Kreitzman \\
\hline $2: 00-2: 30 \mathrm{pm}$ & $\beta$-detected NMR at TRIUMF: Status and Progress & Andrew MacFarlane \\
\hline $2: 30-3: 30 \mathrm{pm}$ & Discussion: Source possibilities, beam profile & \\
\hline $3: 30 \mathrm{pm}-4: 00 \mathrm{pm}$ & Break / Group Photo & \\
\hline $4: 00-5: 30 \mathrm{pm}$ & Discussion: Targets and beamlines & \\
\hline $6: 00-8: 00 \mathrm{pm}$ & Dinner "History of ORNL" & Steve Nagler \\
\hline
\end{tabular}




\section{Future Muon Source Possibilities at the SNS Workshop September 1-2, 2016}

Friday, September 2, 2016

Clinch River Cabin

\begin{tabular}{|c|c|c|}
\hline Time & Event & Speaker \\
\hline 9:00-9:30 am & $\begin{array}{l}\text { First-Order Magnetic Quantum Phase Transitions in Mott } \\
\text { Insulators and Unconventional Superconductors }\end{array}$ & Tomo Uemura \\
\hline 9:30-9:50 am & Science with $\mu$ SR: Hard Condensed Matter & Jeff Sonier \\
\hline 9:50-10:10 am & Magnetism in $\alpha-\mathrm{NaxMnO} 2$ & Michael Graf \\
\hline 10:10-10:30 am & $\begin{array}{l}\text { Muon Spin Rotation/Relaxation as a Probe of } \\
\text { Unconventional Superconductivity }\end{array}$ & Graeme Luke \\
\hline 10:30-10:50 am & Break & \\
\hline 10:50-11:20 am & $\begin{array}{l}\text { Using Muons to Learn about Fe-based Superconductors } \\
\text { and Novel Magnets }\end{array}$ & Stephen Blundell \\
\hline $11: 20-11: 40 \mathrm{am}$ & $\mu \mathrm{SR}$ and $\beta$-NMR of soft matter and chemical systems & Iain McKenzie \\
\hline $11: 40-12: 00 \mathrm{pm}$ & $\mu \mathrm{SR} \&$ Semiconductors & Ross Carroll \\
\hline 12:00-1:00 pm & Lunch & \\
\hline 1:00-2:00 pm & Discussion: Science drivers and Future Plans & \\
\hline $2: 00-2: 15 \mathrm{pm}$ & Closing Remarks & Greg MacDougall \\
\hline $2: 30-4: 00 \mathrm{pm}$ & SNS Tour (Optional) & \\
\hline
\end{tabular}




\section{APPENDIX C. COMPARISON OF EXISTING MUON SOURCES WORLDWIDE}

\begin{tabular}{|l|c|c|c|c|}
\hline \multicolumn{1}{|c|}{ Facility } & Source Type & Flux $\left(\boldsymbol{\mu}^{+} / \mathbf{s e c}\right)^{*}$ & Pulse Width (ns) & Beamlines $^{\S}$ \\
\hline ISIS & Pulsed & $1.5 \times 10^{6}$ & 70 & 4 decay \\
\hline \multirow{2}{*}{ J-PARC } & Pulsed & $1.8 \times 10^{6}$ & 50 & 2 surface / 1 decay / \\
& Continuous & $7.0 \times 10^{4}$ & - & 4 surface / 1 decay \\
\hline PSI & Continuous & $5.0 \times 10^{6}$ & - & 3 surface / 1 decay \\
\hline TRIUMF & Pulsed & $1.66 \times 10^{6}$ & 50 & 4 surface / 1-2 decay \\
\hline Proposed at the SNS &
\end{tabular}

\begin{tabular}{|l|c|c|c|c|}
\hline Facility & Temperature Range & Max Field & Max Pressure & Co-located neutrons? \\
\hline ISIS & & & & Yes \\
\hline J-PARC & & & & Yes \\
\hline PSI & $0.01-1000 \mathrm{~K}$ & $9.5 \mathrm{~T}$ & & Yes \\
\hline TRIUMF & $0.02-900 \mathrm{~K}$ & $9.0 \mathrm{~T}$ & $0.5 \mathrm{GPa}$ & No \\
\hline Proposed at the SNS & - & - & - & Yes \\
\hline
\end{tabular}

* Flux represents the time-averaged flux per instrument, averaged across the facility. Note that continuous sources operating in time-differential mode (measuring one muon at a time) are thus ratelimited to $7.0 \times 10^{4} \mu^{+} / \mathrm{sec}$.

$\S$ Of the currently-constructed beamlines, the decay muon beamline and one of the surface muon beamlines at TRIUMF, as well as the decay muon beamline at J-PARC, are not currently operational. 


\title{
APPENDIX D. INVITATION LETTER TO EXTERNAL PARTICIPANTS
}

\author{
Dear Dr. Williams,
}

Oak Ridge National Laboratory is a world-leading center of neutron science, with excellent infrastructure for materials research, robust facility user programs, and a broad portfolio of science in related areas. At the present time a project is underway to develop a conceptual design for a second target station at the Spallation Neutron Source (SNS). This project would also involve upgrades to the proton accelerator complex. It is now timely to discuss additional possibilities for enhancing the effectiveness of the facility, and to this end we wish to carefully consider whether it makes sense scientifically and technically to utilize protons that would otherwise be directed to a beam dump to power a $\mu \mathrm{SR}, \beta-\mathrm{NMR}$ or related facility. If feasible such a facility would be complementary to the neutron science programs and enhance the overall user programs in materials research.

In order to assess the scientific and technical value of such a facility, we are inviting you, along with other $\mu$ SR scientists, users and beamline staff, to a workshop at Oak Ridge National Laboratory. The aim of this workshop will be to discuss the scientific challenges that can be addressed by neutrons together with a muon / ion source, the technical requirements for building such a facility, and the perspective of the $\mu \mathrm{SR}$ user community on their desire for such a source. The product of this workshop will be a Report, which will include recommendations for the feasibility and value of a muon / ion source and whether provision should be made now for developing such a source in the future. This Report will be shared with the leadership of Oak Ridge National Laboratory and/or the Department of Energy.

We are currently considering holding this workshop Thursday, August $18^{\text {th }}$ to Friday, August $19^{\text {th }}$, 2016, or Thursday, September $1^{\text {st }}$ to Friday, September $2^{\text {nd }}$. Please let us know your willingness to participate and if you are available for either, both or neither of these dates. The dates will be finalized soon based on participants' availability. There will be limited funding available to offset at least part of the travel and accommodation costs for invited participants.

If you have any questions, please feel free to contact either of us and we would be happy to help. We look forward to hearing from you and hopefully welcoming you to Oak Ridge National Laboratory for this workshop.

Sincerely,

Travis Williams

Eugene P. Wigner Fellow

Quantum Condensed Matter Division

Oak Ridge National Laboratory

williamstj@ornl.gov
Gregory MacDougall

Assistant Professor

Department of Physics

University of Illinois at Urbana-Champaign

gmacdoug@illinois.edu 


\section{REFERENCES}

${ }^{7}$ Danilov et al. Phys Rev. STAB. 10, 053501 (2007).

${ }^{8}$ Y. Liu. Private Communication.

${ }^{9}$ S. Kreitzman. Private Communication. 